\title{
A continuum model for flow induced by metachronal coordination between beating cilia
}

\author{
Jeanette Hussong, Wim-Paul Breugem $\dagger$ \\ and Jerry Westerweel \\ Laboratory for Aero and Hydrodynamics, Delft University of Technology, Leeghwaterstraat 21, \\ 2628 CA Delft, The Netherlands
}

(Received 17 August 2010; revised 19 May 2011; accepted 29 June 2011; first published online 30 August 2011)

In this numerical study we investigate the flow induced by metachronal coordination between beating cilia arranged in a densely packed layer by means of a continuum model. The continuum approach allows us to treat the problem as two-dimensional as well as stationary, in a reference frame moving with the speed of the metachronal wave. The model is used as a computationally efficient design tool to investigate ciliainduced transport of a Newtonian fluid in a plane channel. Contrary to prior continuum models, the present approach accounts for spatial variations in the porosity along the metachronal wave and thus ensures conservation of mass within the cilia layer. Using porous-media theory the governing volume-averaged Navier-Stokes (VANS) equations are derived and closure formulations are given explicitly for the model. This makes it possible to investigate cilia-induced flow with a continuum model in both the viscous regime and the inertial regime. The results show that metachronal coordination can act as a transport mechanism in both regimes. Porosity variations appear to be the key mechanism for correct prediction of the fluid transport in the viscous flow regime. The reason is that spatial variations in the porosity break the symmetry of the drag distribution along the metachronal wave. A new insight that has been gained is that the fluid transport reverses, thus switches from plectic to antiplectic metachronism, for the same cilia beat cycle when the wavespeed is increased such that inertial effects occur. Based on a parameter study, the net transport in the channel is described by a power-law relation of the amplitude, length and speed of the metachronal wave. It is found that the wavelength has the strongest effect on the viscosity-dominated fluid transport.

Key words: microfluidics, propulsion

\section{Introduction}

Cilia are slender 5-10 $\mu \mathrm{m}$ long hair-like organelles extending from the cell body. Cilia play a vital role in many biological systems. There are two types of cilia: motile and non-motile. The latter typically function as sensory organelles. Motile cilia in mammalian airways transport mucus and pollution out of the lungs. Various micro-organisms use motile cilia, covering their surface, to propel themselves in water.

$\dagger$ Email address for correspondence: W.P.Breugem@tudelft.nl 
(a)

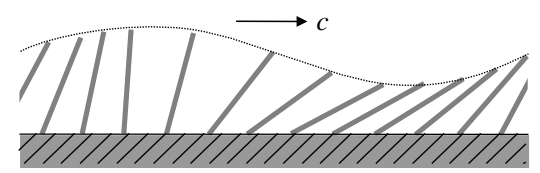

(b) Symmetry plane

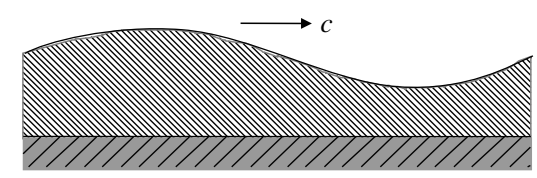

FIGURE 1. (a) Array of cilia attached to both sides of a plane channel. The cilia beat in metachronal coordination which induces surface waves that travel with speed $c$. (b) Array of cilia represented by a periodically deforming porous layer.

Motile cilia typically perform a configurationally asymmetric cilia beat cycle (Khaderi et al. 2010) by carrying out either an oar-like beat cycle or a plain beat cycle, where the cilium stretches during the forward stroke and bends during the backward stroke. A configurationally asymmetric cilia beat cycle is known to be a key mechanism for creating net transport of viscous flows (e.g. Purcell 1977).

When cilia occur in large groups they typically beat slightly out of phase with respect to adjacent cilia. This synchronization between beating cilia is called metachronal coordination. It results in a wave that travels along the cilia tip surface, which we refer to as a metachronal wave.

Inspired by cilia in nature, increased effort is now devoted to the design and fabrication of artificial cilia for microfluidic applications. Numerical studies have been performed by Khatavkar et al. (2007) and Khaderi et al. (2009), showing that electromagnetically driven artificial cilia can induce mixing and pumping. Cilia-like structures of micrometre scale have been built by different groups (see Nonaka et al. 2005; Singh, Laibinis \& Hatton 2005), but it was only recently that artificial cilia could be synthesized and externally actuated to manipulate fluid on sub-millimetre scales (see den Toonder et al. 2007; Baltussen et al. 2009; Fahrni, Prins \& van Ijzendoorn 2009; Oh et al. 2009; Hussong et al. 2011). A brief overview of experimental work is given by Zhou \& Liu (2008). To date, systems with artificial cilia are based on a synchronous cilia motion (see Shields et al. 2010; Vilfan et al. 2009). If cilia beat in synchrony, a strongly oscillating forward-backward flow is induced in the viscous regime and net fluid transport can be achieved only if the cilia beat cycle exhibits a configurationally asymmetric beat cycle.

In this paper we show that metachronal coordination can be used in the design of artificial cilia as another means for inducing net fluid transport. The present paper introduces a numerical continuum model in which an array of out-of-phase beating cilia (figure $1 a$ ) is represented by a periodically deforming porous layer that forms travelling waves of speed $c$ on the surface (figure $1 b$ ). The continuum model serves as a computationally efficient design tool to investigate fluid transport by metachronal coordination between beating cilia. The cilia are considered to be straight cylinders that cover both channel walls. The rigid cilia rotate periodically forward and backward around their base point. The cilia beat is configurationally symmetric since the forward stroke is exactly the opposite of the backward stroke. It means that a net fluid transport can originate solely from the metachronal coordination between beating cilia.

Existing models of cilia-induced flow can be divided into three groups: the discrete cilia models, the cilia envelope models and the cilia sublayer models. In the group of discrete cilia models, the cilia-interaction models, the effect of the cilia on the fluid is represented by a simple viscous drag relation. Such an approach was used by 
Gueron \& Liron (1993), who developed a slender-body approach to simulating threedimensional ciliary beats that allows for cilia interactions. Cilia-interaction models have been extensively used to show that the onset and self-sustaining character of metachronal coordination is the result of hydrodynamic interaction between beating cilia (Gueron et al. 1997; Gueron \& Levit-Gurevich 1998; Lenz \& Ryskin 2006; Guirao \& Joanny 2007). Discrete cilia models that simulate the flow field around the cilia typically make use of the immersed boundary method (IBM: Peskin 1972), in which the effect of cilia on the flow is included as an additional forcing term in the momentum equations. Dauptain, Favier \& Bottaro (2008) developed a threedimensional, one-way coupled cilia model to investigate the flow induced by cilia rows of Pleurobrachia. The flow was in the inertial regime with $50<R e<200$ where the Reynolds number is based on the cilia tip velocity and cilia length. Imposing the cilia velocities obtained from PTV measurements (Barlow \& Sleigh 1993), they found that the propulsion of Pleurobrachia is related to a blowing/suction effect of the system and the beat parameters. Heys et al. (2008) studied ciliary interaction of sensing hairs of crickets by using the penalty immersed boundary method of Kim \& Peskin (2007) to account for inertia and gravity forces of the hairs. Mitran (2007) developed an overlapping grid model in which a moving grid was fitted around single cilia moving over a fixed Cartesian background grid.

Discrete models provide detailed insight into the flow, but they are computationally expensive. They are therefore unsuitable for simulating many cilia or performing extensive parameter studies. When the detailed flow around individual cilia is not of interest, computationally efficient approaches are advantageous such as the envelope and sublayer models. In the envelope model the tip surface of densely packed cilia is approximated as a deforming impermeable wall. Envelope models were extensively used for studies of self-propulsion of minute bodies in a viscous fluid (Taylor 1951; Brennen 1974; Katz 1974) and investigations of the role of transverse and longitudinal wave oscillations (Tuck et al. 1968; Nielsen \& Larsen 1993). Such approaches hinge on the assumption of an impermeable wall, which disregards the inflow and outflow of fluid through a ciliated surface.

The sublayer models represent a ciliated surface as a fully submerged layer of beating structures. Two types of sublayer models can be found in literature, known as the discrete sublayer models and the continuum sublayer models. In the following, we will simply refer to the continuum sublayer models as the continuum models. The discrete sublayer model is based on the slender-body theory for Stokes flow, in which a cilium is represented by force singularities distributed along the cilium's centre line. The velocity profile of cilia beating in metachronism in a viscous fluid is derived by super-positioning the flow induced by multiple cilia (Blake 1972; Liron \& Mochon 1976; Liron 1978; Smith, Gaffney \& Blake 2007b). The discrete sublayer model is only valid for Stokes flow, and since it neglects the hydrodynamic interaction between adjacent cilia it is more appropriate for systems with widely spread cilia (Brennen \& Winet 1977).

In the continuum model the action of cilia is represented by a spatially continuous volume force. Continuum models have often been used to simulate cilia-induced mucus transport and are therefore also called traction-layer models. A forerunner model was developed by Barton \& Raynor (1967), who achieved an analytical continuum formulation by averaging the velocity fields of multiple cilia. Different continuum models have been developed to simulate mucociliary transport of Newtonian and non-Newtonian fluids (Blake \& Winet 1980; King, Agarwal \& Shukla 1993; Smith, Gaffney \& Blake 2007a). The long list of cilia-mucus models 
is thoroughly reviewed by Smith, Gaffney \& Blake (2008). The continuum models mentioned above bear a strong resemblance to the modelling of a so-called Brinkman layer of slow non-uniform flow through a porous medium. They assume a linear relation between cilia-induced drag and velocities in the viscous flow regime. They also neglect variations of porosity along the metachronal wave, thereby violating local mass conservation in the cilia layer.

In the continuum model presented in this paper, the action of the submerged cilia is modelled as a periodically deforming porous layer. Unlike previous continuum models, the model accounts for spatial variations in the porosity along the metachronal wave and ensures conservation of mass within the porous layer. The model makes use of the porous-media theory developed by Whitaker (1999) and others, incorporating the full volume-averaged Navier-Stokes equations, which will be referred to as the VANS equations. We solve the VANS equations in a frame of reference that moves along with the travelling wave, so that the position of the cilia tip surface is fixed in time and the problem can be treated as stationary. For solving the VANS equations appropriate closures are required, for example, for the drag force, which is discussed in $\S 2.1$. The present model is the first continuum model that allows us to investigate cilia-induced flows both in the viscous and inertial regime up to moderate Reynolds numbers. This is of great importance for the process of designing artificial cilia of variable size, interciliary spacing and beat frequencies. To support the results of the present continuum model, we qualitatively compare its velocity field to that of a discrete two-dimensional model. Subsequently, a sensitivity study will reveal the influence of porosity variations on the fluid transport through the effect of mass conservation and the drag force distribution along the metachronal wave. Finally, the influence of the wave amplitude, the wavelength and the wavespeed is investigated. Our study sheds light on the role of metachronal coordination on fluid transport and it reveals the main mechanisms that are responsible for net fluid transport along with or against the direction of the wavespeed.

\section{The volume-averaged Navier-Stokes equations for a deforming porous medium}

In this paper, a dense array of cilia is interpreted as a locally nearly ordered porous layer that is periodically deforming in time. The mathematical framework for describing the macroscopic flow through the porous layer is provided by the method of local volume averaging (Whitaker 1999). In this section, the governing VANS equations are derived and appropriate closures are formulated for e.g. the drag force. The superficial volume average of an arbitrary microscopic quantity $\psi_{\alpha}$ in phase $\alpha$ can be formally defined as (Quintard \& Whitaker 1994):

$$
\left\langle\psi_{\alpha}\right\rangle \equiv \int_{V} m(\boldsymbol{y}) \gamma_{\alpha}(\boldsymbol{x}+\boldsymbol{y}) \psi_{\alpha}(\boldsymbol{x}+\boldsymbol{y}) \mathrm{d} V,
$$

where $\boldsymbol{y}$ is the relative position vector with respect to the centroid $\boldsymbol{x}$ of the averaging volume $V, m$ is the weighting function and $\gamma_{\alpha}$ is the phase-indicator function of phase $\alpha\left(\gamma_{\alpha}=1\right.$ inside phase $\alpha$ and $\gamma_{\alpha}=0$ otherwise). Henceforth $\alpha=\sigma$ denotes the solid phase of a porous medium and $\alpha=\beta$ refers to the fluid phase. Note that $\gamma_{\alpha}$ depends on time for deformable porous media.

To derive a convenient form of the Navier-Stokes equations we need to link volume-averaged derivatives to derivatives of volume-averaged quantities. The general transport theorem provides such a link for time derivatives, which reads for a 
variable $\gamma_{\alpha}$ (Whitaker 1999):

$$
\left\langle\frac{\partial \psi_{\alpha}}{\partial t}\right\rangle=\frac{\partial}{\partial t}\left\langle\psi_{\alpha}\right\rangle-\int_{A_{\beta \sigma}} m(\boldsymbol{y}) \psi_{\alpha}(\boldsymbol{x}+\boldsymbol{y}) \boldsymbol{w} \cdot \boldsymbol{n}_{\beta \sigma} \mathrm{d} A,
$$

where $\boldsymbol{w}$ is the local velocity of the interface, which in our case is equal to the local value of $\boldsymbol{u}_{\beta} . A_{\beta \sigma}$ is the fluid-solid surface area within the averaging volume and $\boldsymbol{n}_{\beta \sigma}$ is the unit normal on $A_{\beta \sigma}$ that points into the solid phase. The spatial averaging theorem relates the volume average of a spatial gradient to the spatial gradient of a volume average (Whitaker 1999):

$$
\left\langle\nabla \psi_{\alpha}\right\rangle=\nabla\left\langle\psi_{\alpha}\right\rangle+\int_{A_{\beta \sigma}} m(\boldsymbol{y}) \psi_{\alpha}(\boldsymbol{x}+\boldsymbol{y}) \boldsymbol{n}_{\beta \sigma} \mathrm{d} A .
$$

Applying volume averaging to the Navier-Stokes equations and using (2.2) and (2.3) yields the volume-averaged Navier-Stokes (VANS) equations:

$$
\begin{gathered}
\nabla \cdot\left\langle\boldsymbol{u}_{\beta}\right\rangle=-\frac{\partial \epsilon_{\beta}}{\partial t} \\
\frac{\partial\left\langle\boldsymbol{u}_{\beta}\right\rangle}{\partial t}+\nabla \cdot\left(\frac{\left\langle\boldsymbol{u}_{\beta}\right\rangle\left\langle\boldsymbol{u}_{\beta}\right\rangle}{\epsilon_{\beta}}\right)+\nabla \cdot\left(\left\langle\boldsymbol{u}_{\beta} \boldsymbol{u}_{\beta}\right\rangle-\frac{\left\langle\boldsymbol{u}_{\beta}\right\rangle\left\langle\boldsymbol{u}_{\beta}\right\rangle}{\epsilon_{\beta}}\right) \\
=-\frac{1}{\rho} \nabla\left\langle p_{\beta}\right\rangle+v \nabla^{2}\left\langle\boldsymbol{u}_{\beta}\right\rangle+v \nabla \cdot \int_{A_{\beta \sigma}} m \boldsymbol{u}_{\beta} \boldsymbol{n}_{\beta \sigma} \mathrm{d} A \\
+\int_{A_{\beta \sigma}} m\left(-\frac{p_{\beta}}{\rho} \boldsymbol{I}+v \nabla \boldsymbol{u}_{\beta}\right) \cdot \boldsymbol{n}_{\beta \sigma} \mathrm{d} A
\end{gathered}
$$

where $\epsilon_{\beta}$ is the volume fraction of the liquid phase and $\epsilon_{\sigma}=1-\epsilon_{\beta}$ is the volume fraction of the solid phase inside $V$. Since $(2.4 b)$ describes the volume-averaged momentum balance of the liquid phase inside a porous medium, it holds for $0<\epsilon \leqslant 1$. The left-hand side of $(2.4 b)$ is expanded by $\nabla \cdot\left(\left\langle\boldsymbol{u}_{\beta}\right\rangle\left\langle\boldsymbol{u}_{\beta}\right\rangle / \epsilon_{\beta}\right)$ such that the third term on the left-hand side represents the effect of subfilter-scale dispersion on transport of volume-averaged momentum. The third term on the right-hand side represents the effect of net transport of solid material across the averaging volume on viscous diffusion of volume-averaged momentum. The last term on the right-hand side represents the drag force that the solid phase exerts on the fluid phase.

To reformulate the VANS equations in a convenient form we make use of intrinsic volume averages. Superficial and intrinsic quantities are linked by the porosity $\epsilon_{\alpha} \equiv\left\langle\gamma_{\alpha}\right\rangle$ of the phase $\alpha$ inside $V$ :

$$
\left\langle\psi_{\alpha}\right\rangle \equiv \epsilon_{\alpha}\left\langle\psi_{\alpha}\right\rangle^{\alpha}
$$

Furthermore we make use of the decomposition of a microscopic quantity $\psi_{\alpha}$ into an intrinsic volume average and a subfilter-scale contribution as introduced by Gray (1975):

$$
\psi_{\alpha}=\left\langle\psi_{\alpha}\right\rangle^{\alpha}+\tilde{\psi}_{\alpha}
$$

An important simplification of the VANS equations can be obtained when assuming that the volume-averaged quantities are well-behaved. A volume-averaged quantity is well-behaved if it undergoes only small changes within an averaging volume. In this case we can make use of the following relation:

$$
\left\langle\left\langle\psi_{\alpha}\right\rangle^{\alpha}\right\rangle^{\alpha} \approx\left\langle\psi_{\alpha}\right\rangle^{\alpha}
$$


The requirement of well-behaved volume-averaged quantities imposes some limitations on the choice of the weighting function and averaging volume, since the flow rapidly changes across the cilia tip interface. Therefore, an appropriate averaging volume for the present study would be a thin elongated slab with a thickness of smaller order than the interface thickness $d_{i}$ and a streamwise extent on the order of the spacing between two neighbouring cilia.

With help of (2.5)-(2.7), we can express the VANS equations $(2.4 a)$ and $(2.4 b)$ in intrinsic quantities:

$$
\begin{gathered}
\nabla \cdot\left\langle\boldsymbol{u}_{\beta}\right\rangle^{\beta}=-\frac{1}{\epsilon_{\beta}}\left(\frac{\partial \epsilon_{\beta}}{\partial t}+\left\langle\boldsymbol{u}_{\beta}\right\rangle^{\beta} \cdot \nabla \epsilon_{\beta}\right), \\
\frac{\partial\left\langle\boldsymbol{u}_{\beta}\right\rangle^{\beta}}{\partial t}+\left\langle\boldsymbol{u}_{\beta}\right\rangle^{\beta} \cdot \nabla\left\langle\boldsymbol{u}_{\beta}\right\rangle^{\beta}+\frac{1}{\epsilon_{\beta}} \nabla \cdot \epsilon_{\beta}\left\langle\tilde{\boldsymbol{u}}_{\beta} \tilde{\boldsymbol{u}}_{\beta}\right\rangle^{\beta} \\
=-\frac{1}{\rho} \nabla\left\langle p_{\beta}\right\rangle^{\beta}+v \nabla^{2}\left\langle\boldsymbol{u}_{\beta}\right\rangle^{\beta}+\frac{v}{\epsilon_{\beta}} \nabla \cdot \int_{A_{\beta \sigma}} m \tilde{\boldsymbol{u}}_{\beta} \boldsymbol{n}_{\beta \sigma} \mathrm{d} A \\
+\frac{1}{\epsilon_{\beta}} \int_{A_{\beta \sigma}} m\left(-\frac{\tilde{p}_{\beta}}{\rho} \boldsymbol{I}+v \nabla \tilde{\boldsymbol{u}}_{\beta}\right) \cdot \boldsymbol{n}_{\beta \sigma} \mathrm{d} A .
\end{gathered}
$$

A detailed derivation of (2.8a) and (2.8b) is given in Appendix A. Equations (2.8a) and $(2.8 b)$ are under-determined due to the unknown subfilter-scale quantities $\tilde{\boldsymbol{u}}_{\beta}$ and $\tilde{p}_{\beta}$. For solving the VANS equations closures are required for the third term on the left-hand side and the last two terms on the right-hand side. This is discussed in the next subsection.

\subsection{Closure formulations}

The third term on the left-hand side of (2.8b) represents the effect of subfilter-scale dispersion. Scaling shows that this term is of lower order of magnitude compared to the drag force and can therefore be neglected (Breugem \& Boersma 2005; Breugem, Boersma \& Uittenbogaard 2006).

To derive a closure model for the third term on the right-hand side of $(2.8 b)$, we assume locally near-uniform motion of the solid phase. This means that the local solid phase velocity can be approximated by its intrinsic average: $\boldsymbol{u}_{\sigma} \approx\left\langle\boldsymbol{u}_{\sigma}\right\rangle^{\sigma}$. This assumption is allowed provided that the streamwise extent of our averaging volume is small compared to the wavelength of the metachronal wave. We can use this assumption to approximate the no-slip condition at the interface $A_{\beta \sigma}$ by

$$
\left\langle\boldsymbol{u}_{\beta}\right\rangle^{\beta}+\tilde{\boldsymbol{u}}_{\beta} \approx\left\langle\boldsymbol{u}_{\sigma}\right\rangle^{\sigma} \quad\left(\text { on } A_{\beta \sigma}\right) .
$$

This relation allows us to approximate the subfilter-scale contribution $\tilde{\boldsymbol{u}}_{\beta}$ by $\tilde{\boldsymbol{u}}_{\beta} \approx$ $\left\langle\boldsymbol{u}_{\sigma}\right\rangle^{\sigma}-\left\langle\boldsymbol{u}_{\beta}\right\rangle^{\beta}$. The third term on the right-hand side of (2.8b) can then be parametrized as follows:

$$
\begin{aligned}
\frac{v}{\epsilon_{\beta}} \nabla \cdot \int_{A_{\beta \sigma}} m \tilde{\boldsymbol{u}}_{\beta} \boldsymbol{n}_{\beta \sigma} \mathrm{d} A & \approx-\frac{v}{\epsilon_{\beta}} \nabla \cdot\left(\left[\left\langle\boldsymbol{u}_{\beta}\right\rangle^{\beta}-\left\langle\boldsymbol{u}_{\sigma}\right\rangle^{\sigma}\right] \int_{A_{\beta \sigma}} m \boldsymbol{n}_{\beta \sigma} \mathrm{d} A\right) \\
& =\frac{v}{\epsilon_{\beta}} \nabla \cdot\left(\left[\left\langle\boldsymbol{u}_{\beta}\right\rangle^{\beta}-\left\langle\boldsymbol{u}_{\sigma}\right\rangle^{\sigma}\right] \nabla \epsilon_{\beta}\right),
\end{aligned}
$$

where we used that $\int_{A_{\beta \sigma}} m \boldsymbol{n}_{\beta \sigma} \mathrm{d} A=-\nabla \epsilon_{\beta}$. This follows from (2.3) by substituting $\psi_{\alpha}=1$. 
For the last term in (2.8b) we make use of the closure formulation proposed by Whitaker (1996) for a rigid porous medium. We assume that this parametrization is also valid for a deforming porous medium where the drag force between the porous medium and the fluid phase is not based on $\left\langle\boldsymbol{u}_{\beta}\right\rangle^{\beta}$ alone but on the difference $\left\langle\boldsymbol{u}_{\beta}\right\rangle^{\beta}-\left\langle\boldsymbol{u}_{\sigma}\right\rangle^{\sigma}$; this is consistent with the Galilean invariance of the drag force and the previous assumption of a locally uniform motion of the solid phase. The closure formulation for the drag force reads:

$$
\frac{1}{\epsilon_{\beta}} \int_{A_{\beta \sigma}} m\left(-\frac{\tilde{p}_{\beta}}{\rho} \boldsymbol{I}+v \nabla \tilde{\boldsymbol{u}}_{\beta}\right) \cdot \boldsymbol{n}_{\beta \sigma} \mathrm{d} A=-v \epsilon_{\beta} \boldsymbol{K}^{-\mathbf{1}}(\boldsymbol{I}+\boldsymbol{F}) \cdot\left[\left\langle\boldsymbol{u}_{\beta}\right\rangle^{\beta}-\left\langle\boldsymbol{u}_{\sigma}\right\rangle^{\sigma}\right],
$$

where $\boldsymbol{I}, \boldsymbol{K}$ and $\boldsymbol{F}$ are the unit, the permeability and the Forchheimer tensors, respectively. In (2.11) the effect of flow non-uniformity on the drag force (Breugem 2007) is neglected, since this correction is usually small; the effective viscosity experienced by the volume-averaged flow is therefore approximated by the fluid viscosity. Neglecting subfilter-scale dispersion and applying (2.10) and (2.11) to (2.8a) and $(2.8 b)$ yields the following closed form of the VANS equations:

$$
\begin{gathered}
\nabla \cdot\left\langle\boldsymbol{u}_{\beta}\right\rangle^{\beta}=-\frac{1}{\epsilon_{\beta}}\left(\frac{\partial \epsilon_{\beta}}{\partial t}+\left\langle\boldsymbol{u}_{\beta}\right\rangle^{\beta} \cdot \nabla \epsilon_{\beta}\right) \\
\frac{\partial\left\langle\boldsymbol{u}_{\beta}\right\rangle^{\beta}}{\partial t}+\left\langle\boldsymbol{u}_{\beta}\right\rangle^{\beta} \cdot \nabla\left\langle\boldsymbol{u}_{\beta}\right\rangle^{\beta}=-\frac{1}{\rho} \nabla\left\langle p_{\beta}\right\rangle^{\beta}+v \nabla^{2}\left\langle\boldsymbol{u}_{\beta}\right\rangle^{\beta}+\frac{v}{\epsilon_{\beta}} \nabla \cdot\left(\left[\left\langle\boldsymbol{u}_{\beta}\right\rangle^{\beta}-\left\langle\boldsymbol{u}_{\sigma}\right\rangle^{\sigma}\right] \nabla \epsilon_{\beta}\right) \\
-v \epsilon_{\beta} \boldsymbol{K}^{-1}(\boldsymbol{I}+\boldsymbol{F}) \cdot\left[\left\langle\boldsymbol{u}_{\beta}\right\rangle^{\beta}-\left\langle\boldsymbol{u}_{\sigma}\right\rangle^{\sigma}\right]
\end{gathered}
$$

where the third and the fourth term on the right-hand side are the closure formulations for the equivalent terms in $(2.12 b)$. The third term on the right-hand side represents the contribution to viscous diffusion of the volume-averaged momentum due to net transport of solid material across the averaging volume. The last term on the righthand side represents the drag force that the solid phase exerts on the fluid phase.

The system of equations given by $(2.12 a)$ and $(2.12 b)$ can now be solved numerically provided that the porosity, the solid phase motion, the permeability and the Forchheimer tensor are given.

\section{The flow geometry}

In this section, we derive expressions for the solid phase motion $\left\langle\boldsymbol{u}_{\sigma}\right\rangle^{\sigma}$, the porosity $\epsilon_{\beta}$, the permeability tensor $\boldsymbol{K}$ and the Forchheimer tensor $\boldsymbol{F}$. The macroscopic flow geometry is illustrated in figure $2(a)$. We consider a plane channel of which the walls are covered by beating cilia in a staggered arrangement. The cilia are considered to be straight rigid cylinders of length $h$ and diameter $D$ which periodically rotate forward and backward around their anchor points between a maximum orientation angle of $\theta_{\max }=\pi / 2$ and a minimum orientation angle of $\theta_{\min }=\arcsin (1-(2 A / h))$ which results from (3.2) and (3.3). Adjacent cilia beat slightly out of phase such that the deviation of the cilia tip surface from its nominal position can be described by a travelling (metachronal) wave of length $\lambda$ and amplitude $A$ that propagates at speed $c$ over the cilia tip surface:

$$
d(x, t)=A \sin (k[x-c t]),
$$

where $A$ is the wave amplitude, $k=2 \pi / \lambda$ the wavenumber with $\lambda$ the wavelength, and $c$ the wavespeed. The orientation angle $\theta$ of the cylindrical cilia elements is related to 
(a)

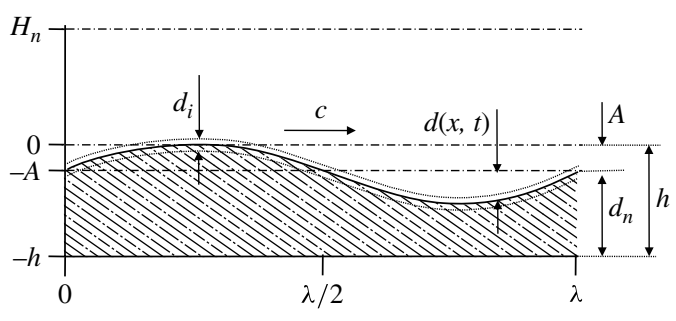

(b)

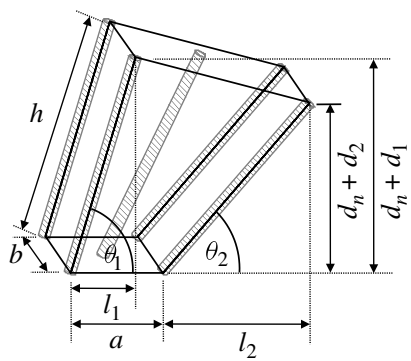

FIGURE 2. (a) Illustration of the densely packed arrangement of cilia attached to the walls of a plane channel. The tip surface of the beating cilia is described by a travelling wave with wave amplitude $A$, wavelength $\lambda$, wavespeed $c$ and interface thickness $d_{i}$. The height of the computational domain equals the sum of the nominal half-channel height $H_{n}$ and the cilia length $h$. (b) Close-up of a unit cell of the staggered cilia arrangement which is used to determine the bulk porosity $\epsilon_{\beta}$. The rigid cilia rotate periodically forward and backward around their base point at $y=-h$. The deviation of the cilia tip position from the nominal thickness of the cilia layer $d_{n}$ at $x \pm a / 2$ is defined as $d_{1,2}=d(x \pm a / 2)$ and consequently $l_{1,2}=\sqrt{h^{2}-\left(d_{1,2}+d_{n}\right)^{2}}$.

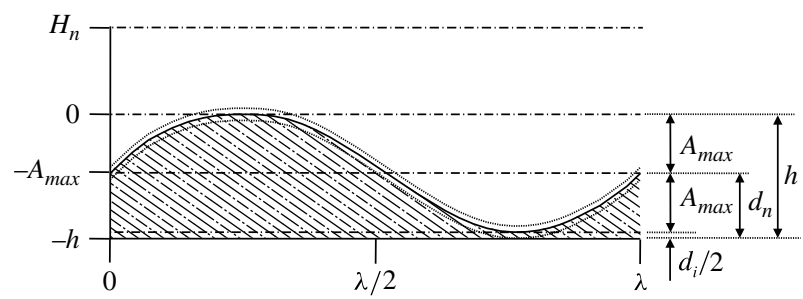

FIgURE 3. The wave amplitude $A$ of the metachronal wave is restricted by the cilia length $h$ and the interface thickness $d_{i}$ of the metachronal wave.

the phase angle of the travelling wave according to

$$
\sin (\theta)=\frac{d+d_{n}}{A+d_{n}}=\left(\frac{A}{h}\right) \sin (k[x-c t])+\left(\frac{d_{n}}{h}\right),
$$

where $d_{n}$ is the nominal thickness of the cilia layer. Since the cilia cannot penetrate the channel wall, the minimum height of the cilia layer equals the cilia thickness. However, to ensure a smooth transition of the porosity at the cilia tip surface, the interface thickness $d_{i}$ must be resolved along the wave (see figure 3 ). The resulting maximum wave amplitude is therefore described by

$$
A_{\max } \leqslant \frac{1}{2}\left(h-\frac{d_{i}}{2}\right)=d_{n}-\frac{d_{i}}{2} .
$$

\subsection{The solid phase motion}

The solid phase velocity $\left\langle\boldsymbol{u}_{\sigma}\right\rangle^{\sigma}$ can be derived from the angular velocity of a cilium which is computed by taking the time derivative of (3.2):

$$
\frac{\partial \theta}{\partial t}=-\frac{A k c \cos (k[x-c t])}{h \cos (\theta)} .
$$


Assuming solid body rotation, the velocity of a small segment of the cylindrical element is given by $\boldsymbol{u}_{\sigma}=[(y+h) / \sin (\theta)](\partial \theta / \partial t) \boldsymbol{e}_{\theta}$, with $\boldsymbol{e}_{\theta}$ being the unit vector pointing in the $\theta$-direction. The horizontal velocity component follows from the inner product with the unit vector $\boldsymbol{e}_{x}$ in the $x$-direction, where $\boldsymbol{e}_{\theta} \cdot \boldsymbol{e}_{x}=-\sin (\theta)$. Assuming locally uniform motion of the solid phase (see $\S 2.1$ ), the solid phase velocity is approximately equal to its intrinsic volume average: $\boldsymbol{u}_{\sigma} \approx\left\langle\boldsymbol{u}_{\sigma}\right\rangle^{\sigma}$. For $h A / \lambda^{2} \ll 1$, the intrinsic solid phase velocities over the height of the cilia layer are well approximated with the velocities along the cilia elements:

$$
\begin{aligned}
& \left\langle u_{\sigma}\right\rangle^{\sigma} \approx A k c \cos [k(x-c t)]\left(\frac{y+h}{d+d_{n}}\right) \tan (\theta), \\
& \left\langle v_{\sigma}\right\rangle^{\sigma} \approx-A k c \cos [k(x-c t)]\left(\frac{y+h}{d+d_{n}}\right) .
\end{aligned}
$$

\subsection{The porosity}

The porosity in the core of the cilia layer is related to the volume fraction of cilia within a representative volume, as shown in figure 2 . For simplicity the porosity is assumed to be only a function of the cilia position in the $x$-direction but constant over the height of the cilia layer, which holds if the ratio of the wave amplitude $A$ to the wavelength $\lambda$ stays small compared to the cilia number density $\lambda / a$, which can be expressed as $A a / \lambda^{2} \ll 1$. After some mathematical manipulations (see Appendix B) the following expression is found for the porosity in the core of the cilia layer:

$$
\epsilon_{\beta, c}(x, t)=1-\frac{\pi D^{2} h}{b\left(a\left[d_{1}+d_{2}+2 d_{n}\right]-l_{1}\left[d_{2}+d_{n}\right]+l_{2}\left[d_{1}+d_{n}\right]\right)},
$$

where $D$ is the cylinder diameter, $b$ is the cilia spacing in the spanwise direction, $d_{1,2}=d(x \pm(1 / 2) a)$ and $l_{1,2}=\sqrt{h^{2}-\left(d_{1,2}+d_{n}\right)^{2}}$.

In a thin layer of thickness $d_{i}$ centred around the cilia tip surface $\left(-(1 / 2) d_{i} \leqslant y \leqslant\right.$ $\left.(1 / 2) d_{i}\right)$, the porosity varies rapidly from $\epsilon_{\beta}=\epsilon_{\beta, c}$ to $\epsilon_{\beta}=1$. A rapid but smooth change in the porosity can be modelled appropriately by a fifth-order polynomial (Breugem \& Boersma 2005):

$$
\begin{aligned}
\epsilon_{\beta, i}(x, y, t)= & 1-6\left(\epsilon_{\beta, c}-1\right)\left(\frac{y+A-d-d_{i} / 2}{d_{i}}\right)^{5}-15\left(\epsilon_{\beta, c}-1\right)\left(\frac{y+A-d-d_{i} / 2}{d_{i}}\right)^{4} \\
& -10\left(\epsilon_{\beta, c}-1\right)\left(\frac{y+A-d-d_{i} / 2}{d_{i}}\right)^{3}
\end{aligned}
$$

The simple porosity formulation (3.6) holds provided that adjacent cilia do not overlap. If it is assumed that $D / h \ll 1$, this requirement is fulfilled when it holds that

$$
\left(\frac{d_{1}+d_{n}}{d_{2}+d_{n}}\right) \geqslant\left(\frac{l_{1}-a}{l_{2}}\right) \text {. }
$$

\subsection{The permeability and Forchheimer tensor}

As a first ansatz for the permeability and Forchheimer tensors we choose the modified Ergun model (MacDonald et al. 1979; Bird, Stewart \& Lightfoot 2002), which is a 
semi-empirical model that is widely used for packed beds:

$$
\begin{gathered}
\boldsymbol{K}=\frac{d_{p}^{2}}{180} \frac{\epsilon_{\beta}^{3}}{\left(1-\epsilon_{\beta}\right)^{2}} \boldsymbol{l}, \\
\boldsymbol{F}=\frac{\epsilon_{\beta}}{100\left(1-\epsilon_{\beta}\right)} \frac{\left|\left\langle\boldsymbol{u}_{\beta}\right\rangle^{\beta}-\left\langle\boldsymbol{u}_{\sigma}\right\rangle^{\sigma}\right| d_{p}}{v} \boldsymbol{I},
\end{gathered}
$$

where $d_{p}$ is the so-called mean particle diameter. In the present model we are dealing with rigid cylinders of diameter $D$, and we therefore use $d_{p}=(3 / 2) D$.

\section{Discretization}

\subsection{The normalized VANS equations}

Henceforth we solve the VANS equations in a frame of reference that moves along with the travelling wave, so that the position of the cilia tip interface is fixed in time and the problem can be treated as stationary. Note that in this case $\partial \epsilon_{\beta} / \partial t=0$.

Our flow geometry contains several velocities and length scales. The bulk velocity in the channel region is expected to scale with the wavespeed $c$, so $c$ is chosen as a characteristic velocity scale. Since the dependency of the bulk velocity on the wavelength and wave amplitude is not known a priori, we simply choose the nominal half-channel height $H_{n}$ as a length scale. The VANS equations are normalized with $c$ and $H_{n}$, hence the Reynolds number reads $R e=H_{n} c / \nu$. In the moving frame of reference the non-dimensionalized VANS equations read:

$$
\begin{aligned}
\nabla \cdot\left\langle\boldsymbol{u}_{\beta}\right\rangle^{\beta}= & -\frac{1}{\epsilon_{\beta}}\left\langle\boldsymbol{u}_{\beta}\right\rangle^{\beta} \cdot \nabla \epsilon_{\beta}, \\
\frac{\partial\left\langle\boldsymbol{u}_{\beta}\right\rangle^{\beta}}{\partial t}= & -\left\langle\boldsymbol{u}_{\beta}\right\rangle^{\beta} \cdot \nabla\left\langle\boldsymbol{u}_{\beta}\right\rangle^{\beta}-\nabla\left\langle p_{\beta}\right\rangle^{\beta}+\frac{1}{R e} \nabla^{2}\left\langle\boldsymbol{u}_{\beta}\right\rangle^{\beta}+\frac{1}{\epsilon_{\beta} R e} \nabla \cdot\left(\left[\left\langle\boldsymbol{u}_{\beta}\right\rangle^{\beta}-\left\langle\boldsymbol{u}_{\sigma}\right\rangle^{\sigma}\right] \nabla \epsilon_{\beta}\right) \\
& -\frac{1}{R e}\left[\frac{\sqrt{180}\left(1-\epsilon_{\beta}\right)}{\epsilon_{\beta} d_{p}}\right]^{2}\left(\left\langle\boldsymbol{u}_{\beta}\right\rangle^{\beta}-\left\langle\boldsymbol{u}_{\sigma}\right\rangle^{\sigma}\right) \\
& -\left[\frac{1.8\left(1-\epsilon_{\beta}\right)}{\epsilon_{\beta} d_{p}}\right]\left|\left\langle\boldsymbol{u}_{\beta}\right\rangle^{\beta}-\left\langle\boldsymbol{u}_{\sigma}\right\rangle^{\sigma}\right|\left(\left\langle\boldsymbol{u}_{\beta}\right\rangle^{\beta}-\left\langle\boldsymbol{u}_{\sigma}\right\rangle^{\sigma}\right) .
\end{aligned}
$$

The last two terms of $(4.1 b)$ were derived by substituting $(3.9 a)$ and $(3.9 b)$ for the permeability and the Forchheimer tensor into the last two terms of $(2.12 b)$, which represent the linear and nonlinear contribution of the drag force that the solid phase exerts on the fluid phase. Note that $(4.1 b)$ is only defined for $0<\epsilon \leqslant 1$. For $\epsilon=1$ it recovers the Navier-Stokes equations. The flow is subjected to the following (nondimensionalized) boundary conditions: $\left\langle u_{\beta}\right\rangle^{\beta}=-1$ and $\left\langle v_{\beta}\right\rangle^{\beta}=0$ at $y / H_{n}=-h / H_{n}$, $\partial\left\langle u_{\beta}\right\rangle^{\beta} / \partial y=\left\langle v_{\beta}\right\rangle^{\beta}=0$ at $y / H_{n}=1$. Periodic boundary conditions are applied at $x=0$ and $x=\lambda$. Note that all results are shown in a fixed frame of reference.

\subsection{Numerical scheme}

The VANS equations $(4.1 a)$ and $(4.1 b)$ are discretized on a staggered and uniform Cartesian grid. In all simulations the dimensions of the grid are $\mathrm{d} x / H_{n}=\mathrm{d} y / H_{n}=$ $1 / 100$. The spatial derivatives are estimated with a second-order central-difference scheme. The flow problem can be treated as stationary in a frame of reference moving along with the travelling wave. A time-marching algorithm is used to reach the stationary solution. The terms in $(4.1 b)$ are integrated in time with a second-order 
Adams-Bashforth scheme, except for the pressure-gradient term that is integrated with the Crank-Nicolson scheme (Wesseling 2001). To satisfy (4.1a), a pressure-correction scheme is used in which the correction pressure is kept small by updating the pressure every time step. The total scheme is second-order accurate in both space and time and can be summarized as follows:

$$
\begin{aligned}
\frac{\hat{u}_{i}-u_{i}^{n}}{\Delta t} & =-\frac{\partial p^{n-1 / 2}}{\partial x_{i}}+\frac{3}{2} R_{i}^{n}-\frac{1}{2} R_{i}^{n-1}, \\
\frac{\partial^{2} \hat{p}}{\partial x_{i}^{2}} & =\frac{1}{\Delta t} \frac{1}{\epsilon_{\beta}} \frac{\partial\left[\epsilon_{\beta} \hat{u}_{i}\right]}{\partial x_{i}}, \\
u_{i}^{n+1} & =\hat{u}_{i}-\Delta t \frac{\partial \hat{p}}{\partial x_{i}}, \\
p^{n+1 / 2} & =p^{n-1 / 2}+\hat{p},
\end{aligned}
$$

where $u_{i}^{n}$ is a short notation for $\left\langle u_{\beta, i}\right\rangle^{\beta}$ at time step $n, \hat{u}_{i}$ is the prediction velocity, $\hat{p}$ is the correction pressure, and $R_{i}$ is the right-hand side of $(4.1 b)$ excluding the pressure-gradient term.

Equation (4.2b) was obtained by taking the divergence of (4.2c), replacing $\partial u_{i}^{n+1} / \partial x_{i}$ by the right-hand side of $(4.1 a)$ and estimating the velocity in this term at time step $n+1$ by $\hat{u}_{i}$. The error in this estimate is small since according to (4.2c) the difference $u_{i}^{n+1}-\hat{u}_{i}$ is given by the product of the time step and the gradient of the correction pressure, which are both kept small in our scheme. For steady flows the error rapidly approaches zero when time progresses. The present scheme allows us to use a fast direct Poisson solver. The VANS equations are solved on an equidistant Cartesian grid with 28500 to 75000 cells for wavelengths between 1.9 and 5.0. The time step $\Delta t$ is computed from the stability constraints for the second-order Adams-Bashforth scheme (Wesseling 2001).

\section{Numerical results}

\subsection{Qualitative comparison with a discrete model}

The macroscopic flow simulated with the continuum model is qualitatively compared with the results of a discrete two-dimensional model. For the discrete model the computationally efficient IBM of Uhlmann (2005) is used. When forcing is applied in a sufficiently smooth spatial field, this method is second-order accurate.

In both models the motion of the cilia tip surface as well as the cilia length, cilia thickness and streamwise spacing in between adjacent cilia are identical and fixed in the simulations. The cilia width in both models differs since the geometry of the discrete model is a two-dimensional configuration of rigid flaps with zero mass flux across the cilia while the continuum model represents a staggered arrangement of straight cylinders where fluid may flow around the cilia. Furthermore, we approximated the cilia layer in the continuum model as an isotropic porous medium by making use of the Ergun model. Due to the different cilia geometry and underlying drag model, the results of the discrete and the continuum model can only be compared qualitatively. The following simulation parameters are the same for all simulations shown in this paper: $h / H_{n}=0.5, D / H_{n}=0.04, a / H_{n}=0.25$ and $d_{i} / H_{n}=0.09$. For comparison a wave amplitude of $A / H_{n}=0.15$ and a wavelength of $\lambda / H_{n}=3$ are chosen (see figure $2 a$ ). 

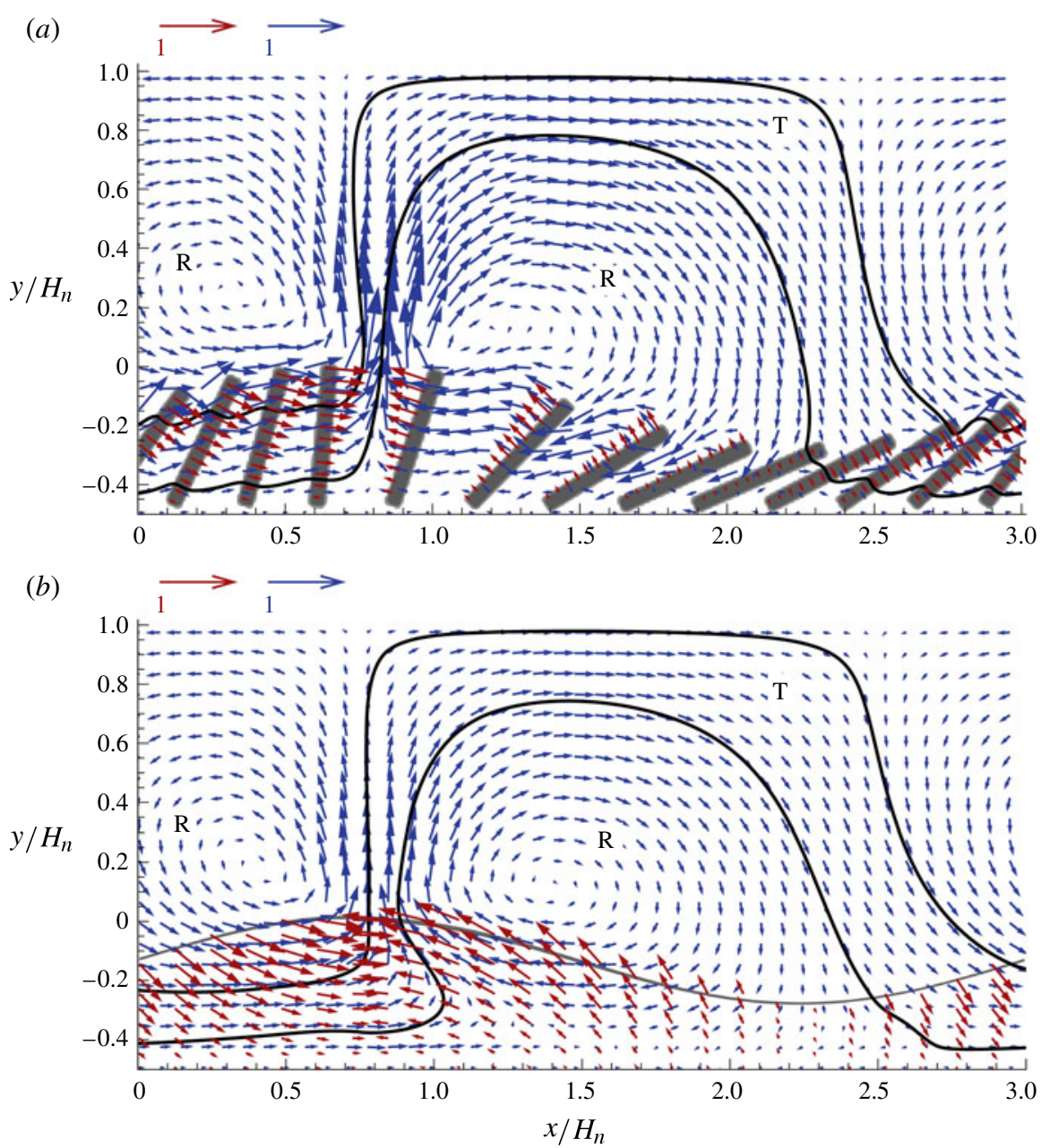

FIGURE 4. Qualitative comparison of the simulated velocity fields at $R e=1$. (a) Discrete IBM model, $(b)$ continuum model.

Results of the discrete and the continuum model are shown in figures 4 and 6 in a fixed frame of reference for a low and a high Reynolds number, respectively. In the results for the discrete model the positions of the cilia are indicated by grey bars. For the continuum model the cilia tip surface is indicated by a grey line. Velocity vectors are shown in blue for the fluid phase and in red for the solid phase. Streamlines are used to border regions of fluid recirculation, which are labelled $\mathrm{R}$, and regions of fluid transport, which are labelled T. For ease of discussion, we define in the following the streamwise fluid velocity as positive when it points in the same direction as the wavespeed and negative otherwise.

\subsubsection{The viscosity-dominated flow regime}

Figure 4 shows that at $R e=1$ the simulated velocity field of the continuum model is very similar to that of the discrete model. We verified that at this Reynolds number the flow is dominated by viscous diffusion, i.e. inertial effects are negligible. A striking feature of the flow is the upward directed fluid jet near the wave top at $x=\lambda / 4$. This 


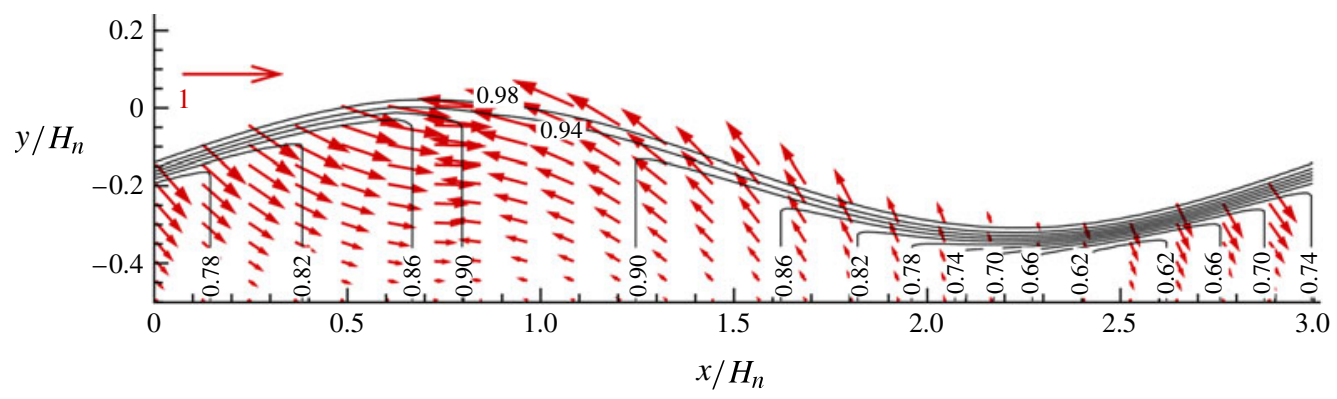

FIGURE 5. Porosity distribution and intrinsic solid phase velocities in the continuum model.

originates from cilia that move towards each other and thereby squeeze fluid out of the voids in between them. At low Reynolds number this results in an upward fluid jet that splits into a clockwise and an anticlockwise rotating recirculation cell. Part of the ejected fluid is deflected in the propagation direction of the wave and dragged into the cilia layer at the lee side of the wave until it merges into the jet of the next wave downstream. The interplay between fluid being ejected and dragged generates a 'street' along which the actual fluid transport takes place. The different transport (T) and recirculation $(\mathrm{R})$ regions are bordered by streamlines.

Figure 5 depicts the porosity distribution inside the cilia layer. From this figure it is observed that the porosity distribution is horizontally asymmetric with respect to a line passing through the wave top at $x / H_{n}=3 / 4$. A lower porosity at the lee side compared to a higher porosity at the front side of the wave results in an asymmetric distribution of the drag force according to the penultimate term in (4.1b). The positive drag force at the lee side of the wave is larger than the negative drag force at the front side of the wave, since the forward motion of the cilia at the lee side coincides with a lower porosity.

\subsubsection{The inertia-dominated flow regime}

Figure $6(a, b)$ shows vector fields of the inertia-dominated flow. For the continuum model the nonlinear drag contribution inside the cilia layer dominates over the linear drag contribution for $R e>1450$. Therefore we used $R e=2000$ for the continuum model to qualitatively compare the results at high Reynolds number. We verified that at $R e=2000$ the nonlinear drag contributes on average approximately $60 \%$ to the total drag inside the cilia layer. It is remarked that in figure 6 the Reynolds number is not the same for the discrete and the continuum model. This is because the Reynolds number only represents the wavespeed and the channel height, while it does not account for the differences in the underlying cilia geometries of the discrete model and the continuum model. Related to the different underlying cilia geometries, inertial effects are much less pronounced in the continuum model, which implies that equal Reynolds numbers in the two models do not yield quantitatively equal flow results. A quantitative validation of the present continuum model requires a comparison with a three-dimensional discrete model of the same underlying geometry (i.e. cylinders in a staggered arrangement). Moreover, such a discrete model can be used to develop and test a closure formulation for the drag force that more closely represents the action of fast-beating cylindrical cilia than the formulation used in the present continuum model as a first ansatz (see §3.3). Such an investigation lies beyond the scope of the present investigation and is left for future studies. Although in the discrete model 


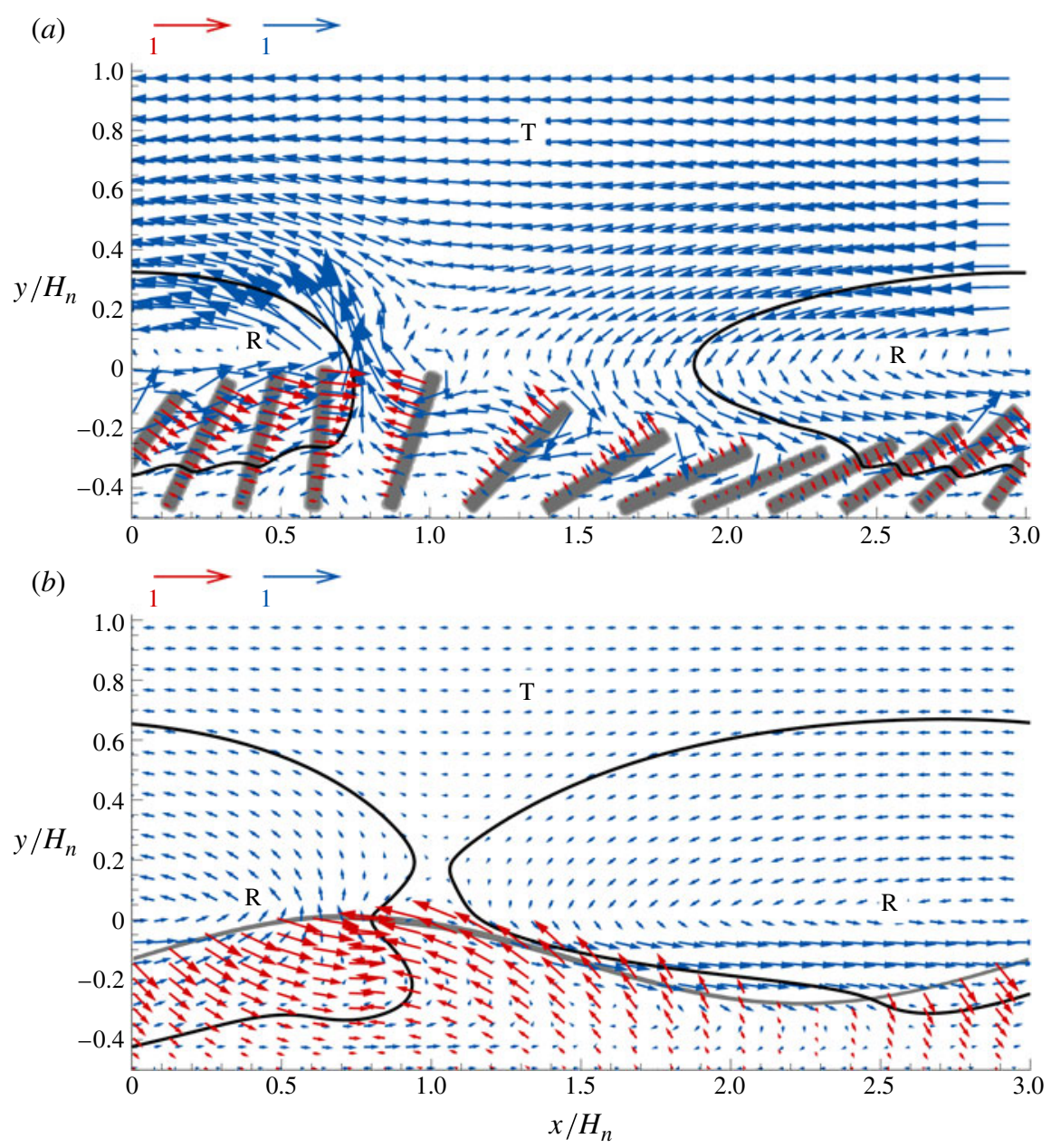

FIGURE 6. Qualitative comparison of the simulated velocity fields. (a) Discrete IBM model at $R e=100$. (b) Continuum model at $R e=2000$.

inertial effects become dominant at a significantly lower Reynolds number than in the continuum model, the changes in the macroscopic flow pattern are qualitatively similar for the transition from a viscosity-dominated to an inertia-dominated flow regime. Unlike the low Reynolds number case, at high Reynolds number the fluid jet near the wave top bends opposite to the direction of wave propagation and merges into a single anticlockwise rotating recirculation cell. The deflection of the jet seems to originate from a low-pressure region at the lee side of the wave. This in turn can be related to the accelerating flow along the streamlines in this region, while simultaneously viscous diffusion appears to be negligible. The recirculation cell extends in the $y$-direction up to about $y / H_{n} \approx 0.5$ and separates a major part of the cilia layer from the fluid in the core of the channel. In the channel core the sense of rotation of the recirculation cell induces a fluid transport in the direction opposite to the wavespeed. 
Vortices at the tip of the cilia are present in the discrete model, for instance at $x / H_{n} \approx 1$ and $x / H_{n} \approx 1.5$. These tip vortices are absent in the continuum model since this model describes only the volume-averaged flow.

\subsection{Sensitivity study of the effect of spatial porosity variations}

The results in the previous section substantiate that metachronal coordination between beating cilia may lead to net fluid transport. It was suggested that spatial variations in the porosity along the cilia layer play a crucial role in this. In the governing equations, the effect of porosity variations become evident in the mass conservation equation (4.1a) and in the drag force formulation in the momentum equation (4.1b). In a sensitivity study we investigated the effect of porosity variations on the flow for $\lambda / H_{n}=3, A / H_{n}=0.15$ and $R e=0.01$. When porosity variations were neglected in the drag force formulation or in the mass conservation equation, a constant porosity value of $\epsilon_{\beta}=0.82$ was chosen, which is close to the spatially averaged porosity value derived from the porosity distribution shown in figure 5. It is noted that the results are qualitatively similar for different uniform porosity values $\left(0.62 \leqslant \epsilon_{\beta} \leqslant 0.92\right)$. The resulting wave-averaged superficial velocity profiles are depicted in figure 7 . The velocity profile including all effects of porosity variations is denoted by $\epsilon_{\beta}(x)$ (solid line without symbols). The velocity profile that results from a constant porosity in both (4.1a) and (4.1b) is labelled $\epsilon_{\beta}=0.82$ (line with triangles). A comparison of both velocity profiles indicates that neglecting porosity variations leads to a strong underestimation of the net flow and even predicts a fluid transport in the reversed direction. A similar reversal in flow direction is observed when porosity variations are neglected only in the drag force formulation $\left(\epsilon_{\beta, d}=0.82\right.$, circles). Neglecting porosity variations in the mass conservation equation, on the other hand, leads to an overestimation of the fluid transport $\left(\epsilon_{\beta, c}=0.82\right.$, squares).

Interestingly, when porosity variations are neglected in both the mass conservation equation and the drag force formulation, the horizontal solid phase velocity does not influence the flow. As depicted in figure 7 the resulting velocity profiles for $\epsilon_{\beta}=0.82$ with $\left\langle u_{\sigma}\right\rangle^{\sigma}=0$ and $\epsilon_{\beta}=0.82$ with $\left\langle u_{\sigma}\right\rangle^{\sigma} \neq 0$ fall on top of each other (lines with triangles and hollow circles). The reason is that for a constant porosity and a configurationally symmetric cilia beat the net horizontal drag force along the metachronal wave is zero. In this case the flow is created by peristaltic motion of the cilia layer.

From the above sensitivity study it is concluded that at low Reynolds numbers the fluid transport is predominantly driven by the effect of porosity variations on the distribution of the drag force along the metachronal wave. However, to correctly predict the flow created by cilia beating in metachronal coordination, porosity variations have to be incorporated in both the drag force formulation and the mass conservation equation.

\subsection{Influence of wave amplitude, length and speed on fluid transport}

A parameter study is performed of the three parameters of the metachronal wave that dominate the flow: the wave amplitude $A$, the wavelength $\lambda$, and the wavespeed $c$. Since the Reynolds number $R e=c H_{n} / v$ is linearly proportional to the wavespeed, we represent an increasing wavespeed by an increasing Reynolds number.

The requirements of non-overlapping cilia and finite thickness of the cilia layer at $x=(3 / 4) \lambda$ impose restrictions on the range of the wave parameters $A$ and $\lambda$ : see (3.3) and (3.8). Based on these criteria the maximum wave amplitude should be $A / H_{n} \lesssim 0.25$ for $\lambda / H_{n}=3.0$ and the wavelength should be $\lambda / H_{n} \gtrsim 1.9$ for 


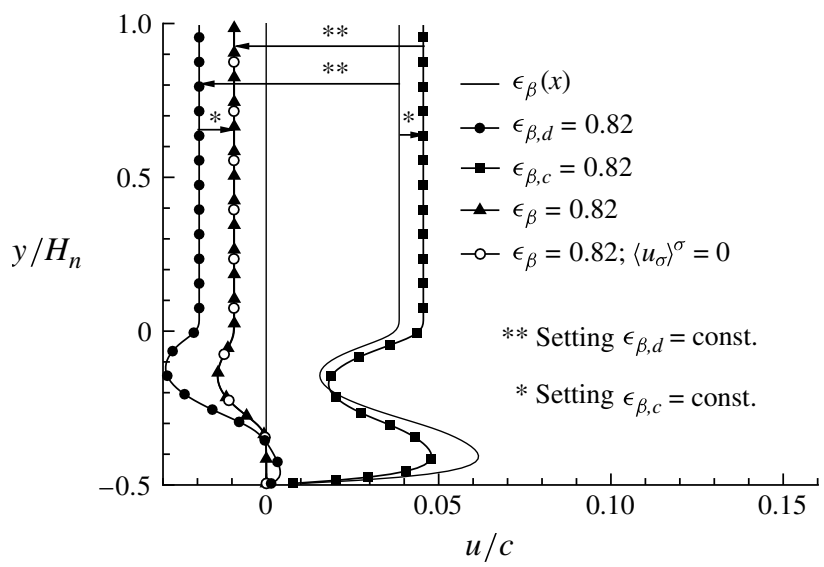

FIGURE 7. Effect of spatial porosity variations on the wave-averaged velocity profile $u(y) / c$. The indices $c$ and $d$ denote a constant porosity in the mass conservation equation (4.1a) and the drag force formulation in the momentum $(4.1 b)$, respectively.
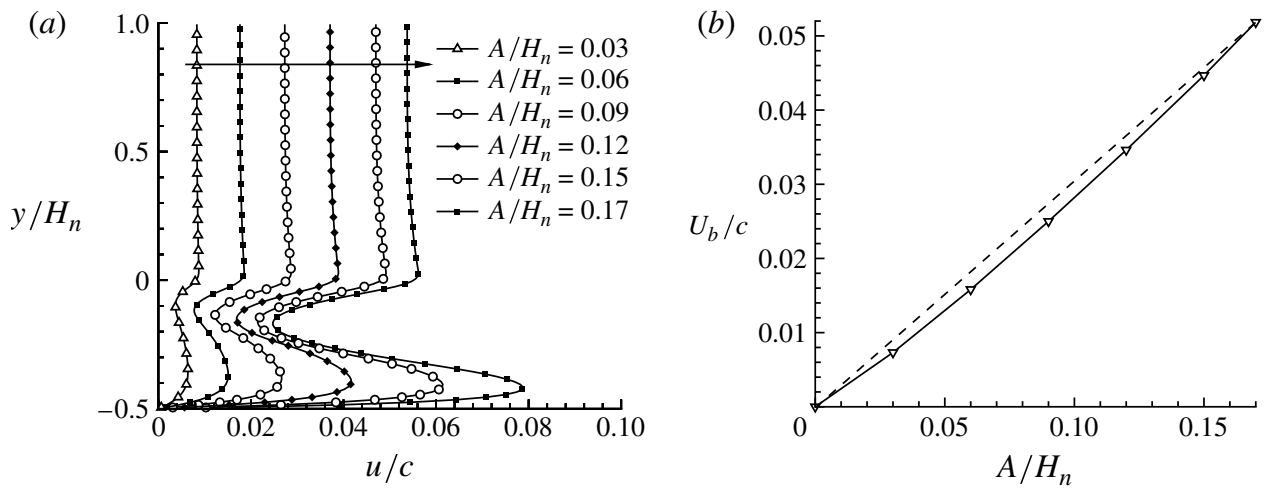

FIGURE 8. Results for increasing wave amplitudes $A / H_{n} \in[0,0.17]$ at $\lambda / H_{n}=3.0$ and $R e=1$. (a) Wave-averaged velocity profiles $u(y) / c$. The arrow points in the direction of increasing $A / H_{n}$. (b) Bulk velocities.

$A / H_{n}=0.15$. The chosen range of wave amplitude and wavelength are well within these limits.

Figures $8(a), 9(a)$ and $10(a)$ show the simulated velocity profiles averaged over one wavelength. Figures $8(b), 9(b)$ and $10(b)$ show the corresponding net flow rates $U_{b} / c=\left(1 / c \lambda\left(h+H_{n}\right)\right) \int_{-h}^{H_{n}} \int_{0}^{\lambda} \epsilon_{\beta} u(x, y) \mathrm{d} x \mathrm{~d} y$. In figure $8(a)$ velocity profiles are shown for increasing wave amplitudes at a fixed wavelength of $\lambda / H_{n}=3.0$ and fixed Reynolds number of $R e=1$. Within the cilia layer $\left(-0.5<y / H_{n}<0\right)$ the waveaveraged velocity is strongly varying. The velocity profile has a local minimum in the upper half and a local maximum in the lower half of the cilia layer. The waveaveraged velocity is nearly constant above the cilia layer $\left(0<y / H_{n}<1\right)$. Figure $8(b)$ shows that the bulk velocity increases almost linearly with the wave amplitude, which is expressed by the following proportionality: $U_{b} / c \sim\left(A / H_{n}\right)^{p}$ with $p=1.127 \pm 0.004$ based on a least-squares error estimate. 

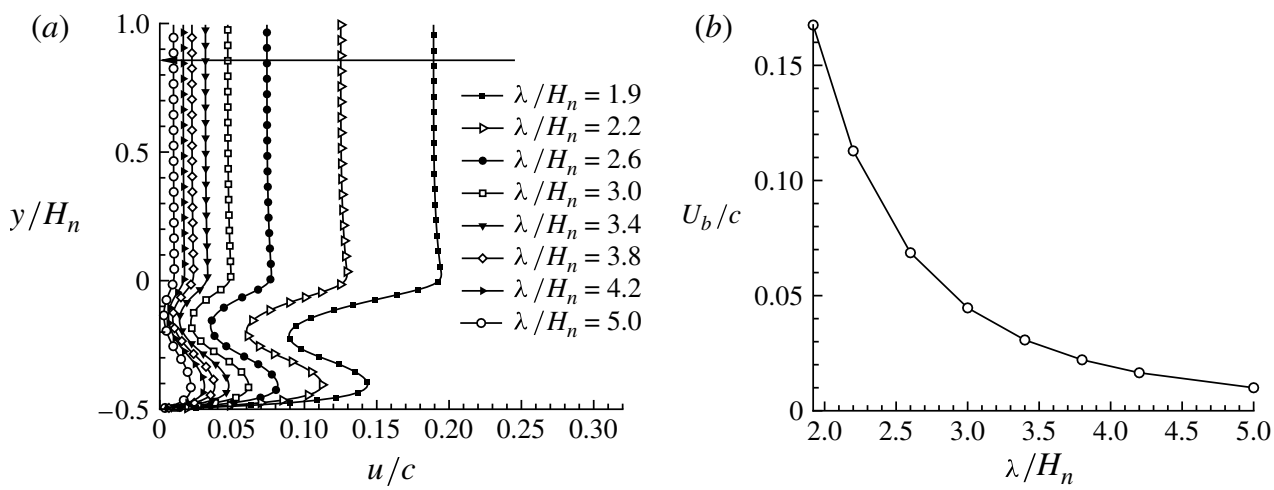

FIGURE 9. Results for increasing wavelengths $\lambda / H_{n} \in[1.9,5.0]$ at $A / H_{n}=0.15$ and $R e=1$. (a) Wave-averaged velocity profiles $u(y) / c$. The arrow points in the direction of increasing $\lambda / H_{n}$. (b) Bulk velocities.
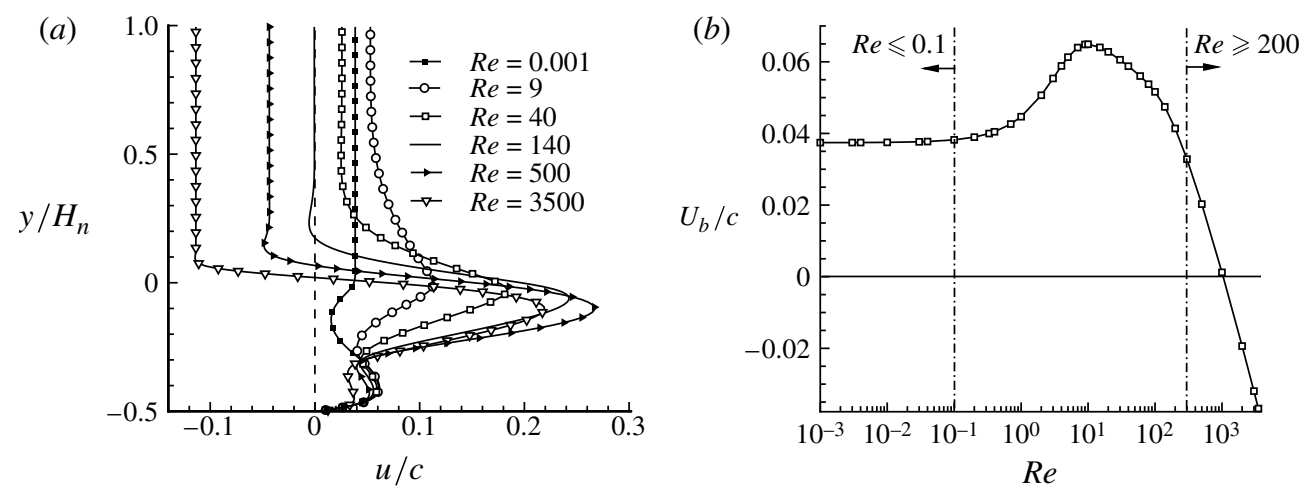

FIGURE 10. Results for increasing Reynolds number $R e=H_{n} c / v \in\left[10^{-3}, 3500\right]$ at $A / H_{n}=0.15$ and $\lambda / H_{n}=2.2$. (a) Velocity profiles $u(y) / c$. (b) Bulk velocities.

Like figure 8, figure 9 shows the effect of the wavelength on the wave-averaged velocity and the bulk velocity. The wave amplitude is fixed at $A / H_{n}=0.15$ and $R e=1$, so the flow is in the viscous regime. In the limit $\lambda / H_{n} \rightarrow \infty$, the net flow rate approaches zero, since in this limit all cilia beat in synchrony and thereby induce a reversing forward-backward fluid motion in the viscous flow regime. A maximum flow rate of $U_{b} / c=0.17$ is reached at wavelength $\lambda / H_{n}=1.9$ (see figure $9 b$ ), which is almost four times higher than the flow rate reached at $\lambda / H_{n}=3$ for the same wave amplitude. Note that $\lambda / H_{n}=1.9$ corresponds to nearly intersecting cilia; further reducing the wavelength is physically prohibited. The bulk velocity decreases with the wavelength according to $U_{b} / c \sim\left(H_{n} / \lambda\right)^{p}$ with $p=2.958 \pm 0.008$.

In figure 10 the effect of the Reynolds number is depicted. The wave amplitude and wavelength are fixed at $A / H_{n}=0.15$ and $\lambda / H_{n}=3.0$, respectively. At small Reynolds numbers, the wave-averaged velocity profile is positive and relatively uniform over the channel height (figure $10 a$ ). The normalized bulk velocity approaches $U_{b} / c \approx 0.037$ for $R e \sim 0$ (figure $10 b$ ), showing that the bulk velocity scales linearly with the wavespeed in the viscous regime. Figure $10(a)$ reveals that for increasing Reynolds 

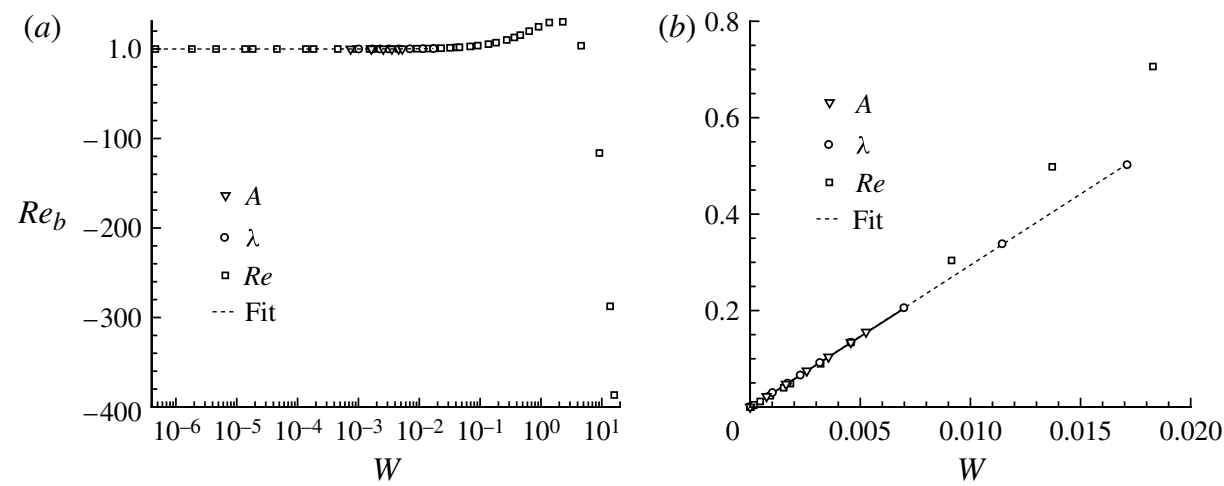

FIGURE 11. Results for the bulk Reynolds number $\operatorname{Re}_{b}$ for varying $W$ (defined in (5.1)). The solid line indicates a linear fit to the data with slope $\alpha=29.49 \pm 0.06$. Different symbols correspond to data points from figures $8(b), 9(b)$ and $10(b)$. (a) Values plotted for full data range. (b) Values plotted for $0<R e_{b}<0.6$.

numbers a positive velocity peak evolves in the velocity profile around $y \approx 0$. At a Reynolds number of $R e \approx 9$ the bulk velocity reaches a maximum (figure $10 b$ ). At this Reynolds number the flow inside the cilia layer is dominated by linear drag (i.e. the last term in $(4.1 b)$ is negligible compared to the penultimate term in $(4.1 b)$ ). When the Reynolds number is further increased the flow in the core of the channel decreases. At $R e \approx 140$ the velocities in the channel core are zero while a positive net fluid transport takes place within the cilia layer. In the Reynolds number range of $200 \lesssim R e \lesssim 3500$ the bulk flow $U_{b} / c$ decreases according to $U_{b} / c \sim \log _{10}\left(R e^{p}\right)$ with $p=-0.064 \pm 0.002$. At a Reynolds number of $R e \approx 1000$ the bulk velocity drops to zero. In this case the positive net flow in the cilia layer balances the negative net flow in the core of the channel (figure 10a). For higher Reynolds numbers the backflow in the channel core increases further while the flow in the cilia layer does not, leading to an overall negative bulk velocity. At a Reynolds number of $R e \approx 1500$ linear and nonlinear drag contribute equally to the transport within the cilia layer. If the Reynolds number is increased beyond $R e \approx 3500$ shear instabilities of the Kelvin-Helmholtz type occur at the lee side of the wave where vertical gradients in the streamwise velocity are large (see figure $6 b$ ). The flow becomes instationary and exhibits travelling waves. The transition to a turbulent flow is suppressed in the present two-dimensional simulations, since vortex stretching is essentially a three-dimensional phenomenon. The investigation of this high-Reynolds-number range at which the flow might become turbulent is beyond the scope of this paper. Therefore only results up to $R e=3500$ are shown in figures 10 and 11.

From figures $8(b), 9(b)$ and $10(b)$, relations have been derived for the bulk velocity as a function of the wave amplitude, length and speed. For the viscous regime these relations can be combined to relate the bulk Reynolds number $\operatorname{Re}_{b} \equiv 2 U_{b}\left(H_{n}+h\right) / v$ to the characteristics of the metachronal wave according to

$$
R e_{b} \equiv \alpha W \quad \text { with } W=\left(\frac{A}{H_{n}}\right)^{1.13}\left(\frac{H_{n}}{\lambda}\right)^{2.96}\left(\frac{c H_{n}}{v}\right),
$$

where $\alpha$ is a constant of proportionality. $W$ is introduced here for convenience and referred to as the viscous wave parameter. Figure $11(a, b)$ shows the bulk Reynolds 
number plotted as a function of the viscous wave parameter $W$. In figure 11(a) all data points of figures $8-10$ are plotted, while figure $11(b)$ shows only values for $0<R e_{b}<0.75$. Up to $R e_{b} \approx 0.2$, the results of figures $8-10$ fall on top of one straight line, which is plotted as a solid line in figure $11(a, b)$. The slope $\alpha$ equals $\alpha=29.49 \pm 0.06$ as obtained from line-fitting according to the least-squares error method. The extrapolation of the best-fit line is shown by a dash-dotted line. For $\operatorname{Re}_{b}>0.2$ the results increasingly deviate from the power-law relation given by (5.1).

\section{Summary and discussion}

In the present study a new continuum model has been presented to investigate flow induced by metachronal coordination between adjacent cilia. The main advantage of the continuum model over a discrete model is its computational efficiency. This is because the two-dimensional continuum model allows us to simulate a staggered arrangement of cylindrical cilia which otherwise would require a three-dimensional discrete model. For simplicity we qualitatively compared the continuum model with a simple discrete model. Despite the geometrical differences between both models, the same macroscopic flow characteristics were observed in the simulation results.

In contrast to existing continuum models (Blake \& Winet 1980; King et al. 1993; Smith et al. 2007a), the presented continuum model considers porosity variations along the metachronal wave and thereby ensures mass conservation within the cilia layer. The modulation of the porosity along the metachronal wave was shown to be an essential feature for the viscous fluid transport. A sensitivity study showed that neglecting porosity variations in the mass conservation equation leads to an overestimation of the net flow while the fluid transport rates can be strongly underestimated and even lead to a reversed transport direction if porosity variations are not considered in the drag force formulation. At low Reynolds numbers, the fluid transport is predominantly driven by the interplay of solid phase velocities and porosity variations present in the drag force formulation. The reason is that the presence of porosity variations breaks the symmetry of the drag distribution along the metachronal wave such that forward moving cilia overlap with a low porosity region creating a positive net drag force that leads to symplectic metachronism. Fluid is transported in the direction of the wavespeed by being continuously ejected from the cilia layer at the wave top and dragged into the cilia layer at the lee side of the next wave.

Existing studies showed that metachronal coordination increases fluid transport if combined with a configurationally asymmetric cilia beat cycle (Khaderi et al. 2010). We show that metachronal coordination can act as independent transport mechanism in the viscous and inertial regime. For the design of artificial cilia it means that, in the viscous regime, any kind of cilia beat can create net flow, if the cilia beat slightly out of phase.

While existing sublayer models are limited to Stokes flow, the present model holds for both small and moderate Reynolds numbers, such that cilia-induced flows within a wide range of metachronal wave parameters can be studied. The parameter study showed that the same type of cilia beat leads to a flow reversal by increasing the wavespeed. When advection of momentum dominates over viscous diffusion the fluid transport is altered by the presence of pressure variations. Fluid is accelerated along the streamlines at the lee side of the wave. This is accompanied by a low-pressure region, which deflects ejected fluid at the wave top in the 
direction opposite to the wavespeed. The jet merges into one single anticlockwise rotating recirculation cell inducing a fluid transport in the direction opposite to the wavespeed. From earlier Stokes flow models it was known that symplectic and antiplectic metachronism depend on the shape asymmetry of the cilia beat (Blake 1972). We show that a switch from symplectic to antiplectic metachronism is possible by increasing the beat frequency. It might shed light on the fact that in nature relatively stiff and straight cilia beat in symplectic metachronism at low Reynolds numbers (Lardner \& Shack 1972), while antiplectic metachronism is often observed at higher Reynolds numbers such as with the paddle worm (Phyllodoce maculata) (Merz \& Edwards 1998) or the sea gooseberry (Pleurobrachia pileus) (Dauptain et al. 2008).

For the specific cilia geometry and beat cycle studied in this paper a power-law relation of the amplitude, length and speed of the metachronal wave is found for the net transport in the channel at low bulk Reynolds numbers (see (5.1)). For the design of artificial cilia we conclude from (5.1) that of all three wave parameters the wavelength has the strongest effect on the viscosity-dominated fluid transport.

This work is part of the European project ARTIC, under Contract No. STRP 033274. We would like to thank Professor J. M. J. den Toonder for his suggestions for improving the manuscript.

\section{Appendix A. The VANS equations in intrinsic quantities}

\section{A.1. The volume-averaged continuity equation}

The volume-averaged continuity equation of the liquid phase $\beta$ is given in $(2.4 a)$ in superficial quantities:

$$
\nabla \cdot\left\langle\boldsymbol{u}_{\beta}\right\rangle=-\frac{\partial \epsilon_{\beta}}{\partial t} .
$$

With (2.5) we can express (A 1) in intrinsic quantities:

$$
\nabla \cdot\left(\epsilon_{\beta}\left\langle\boldsymbol{u}_{\beta}\right\rangle^{\beta}\right)=-\frac{\partial \epsilon_{\beta}}{\partial t} .
$$

By applying the chain rule to the left-hand side term in (A 2) and dividing by the porosity, $(2.4 a)$ is readily obtained:

$$
\nabla \cdot\left\langle\boldsymbol{u}_{\beta}\right\rangle^{\beta}=-\frac{1}{\epsilon_{\beta}}\left[\frac{\partial \epsilon_{\beta}}{\partial t}+\left\langle\boldsymbol{u}_{\beta}\right\rangle^{\beta} \cdot \nabla \epsilon_{\beta}\right] .
$$




\section{A.2. The volume-averaged momentum equation}

Accordingly, we derive the volume-averaged momentum equations in intrinsic quantities starting from $(2.4 b)$ :

$$
\begin{aligned}
\frac{\partial\left\langle\boldsymbol{u}_{\beta}\right\rangle}{\partial t}+\nabla \cdot\left(\frac{\left\langle\boldsymbol{u}_{\beta}\right\rangle\left\langle\boldsymbol{u}_{\beta}\right\rangle}{\epsilon_{\beta}}\right)+\nabla \cdot\left(\left\langle\boldsymbol{u}_{\beta} \boldsymbol{u}_{\beta}\right\rangle-\frac{\left\langle\boldsymbol{u}_{\beta}\right\rangle\left\langle\boldsymbol{u}_{\beta}\right\rangle}{\epsilon_{\beta}}\right) \\
=-\frac{1}{\rho} \nabla\left\langle p_{\beta}\right\rangle+v \nabla^{2}\left\langle\boldsymbol{u}_{\beta}\right\rangle+v \nabla \cdot \int_{A_{\beta \sigma}} m \boldsymbol{u}_{\beta} \boldsymbol{n}_{\beta \sigma} \mathrm{d} A \\
\quad+\int_{A_{\beta \sigma}} m\left(-\frac{p_{\beta}}{\rho} \boldsymbol{I}+v \nabla \boldsymbol{u}_{\beta}\right) \cdot \boldsymbol{n}_{\beta \sigma} \mathrm{d} A
\end{aligned}
$$

For ease of reading, each term in (A $4 a$ ) is treated separately in the following. The first term on the left-hand side of (A $4 a$ ) is rewritten in intrinsic quantities and decomposed by making use of the chain rule:

$$
\frac{\partial\left\langle\boldsymbol{u}_{\beta}\right\rangle}{\partial t}=\frac{\partial}{\partial t}\left(\epsilon_{\beta}\left\langle\boldsymbol{u}_{\beta}\right\rangle^{\beta}\right)=\left\langle\boldsymbol{u}_{\beta}\right\rangle^{\beta} \frac{\partial \epsilon_{\beta}}{\partial t}+\epsilon_{\beta} \frac{\partial\left\langle\boldsymbol{u}_{\beta}\right\rangle^{\beta}}{\partial t} .
$$

The second and the last term on the left-hand side of (A $4 a$ ) are expressed in intrinsic quantities according to (2.5):

$$
\nabla \cdot\left(\frac{\left\langle\boldsymbol{u}_{\beta}\right\rangle\left\langle\boldsymbol{u}_{\beta}\right\rangle}{\epsilon_{\beta}}\right)=\nabla \cdot\left(\frac{\epsilon_{\beta}\left\langle\boldsymbol{u}_{\beta}\right\rangle^{\beta} \epsilon_{\beta}\left\langle\boldsymbol{u}_{\beta}\right\rangle^{\beta}}{\epsilon_{\beta}}\right)=\nabla \cdot\left(\epsilon_{\beta}\left\langle\boldsymbol{u}_{\beta}\right\rangle^{\beta}\left\langle\boldsymbol{u}_{\beta}\right\rangle^{\beta}\right) .
$$

The second term on the left-hand side of (A $4 a$ ) is further rearranged by applying the chain rule to (A 6):

$$
\nabla \cdot\left(\epsilon_{\beta}\left\langle\boldsymbol{u}_{\beta}\right\rangle^{\beta}\left\langle\boldsymbol{u}_{\beta}\right\rangle^{\beta}\right)=\left\langle\boldsymbol{u}_{\beta}\right\rangle^{\beta} \nabla \cdot\left\langle\boldsymbol{u}_{\beta}\right\rangle+\epsilon_{\beta}\left\langle\boldsymbol{u}_{\beta}\right\rangle^{\beta} \cdot \nabla\left\langle\boldsymbol{u}_{\beta}\right\rangle^{\beta} .
$$

The third term on the left-hand side of (A $4 a$ ) is reformulated in intrinsic quantities with (2.5) and hence decomposed according to Gray's decomposition (2.6):

$$
\begin{aligned}
\nabla \cdot\left\langle\boldsymbol{u}_{\beta} \boldsymbol{u}_{\beta}\right\rangle & =\nabla \cdot\left(\epsilon_{\beta}\left\langle\boldsymbol{u}_{\beta} \boldsymbol{u}_{\beta}\right\rangle^{\beta}\right)=\nabla \cdot\left(\epsilon_{\beta}\left\langle\left(\left\langle\boldsymbol{u}_{\beta}\right\rangle^{\beta}+\tilde{\boldsymbol{u}}_{\beta}\right)\left(\left\langle\boldsymbol{u}_{\beta}\right\rangle^{\beta}+\tilde{\boldsymbol{u}}_{\beta}\right)\right\rangle^{\beta}\right) \\
& =\nabla \cdot\left(\epsilon_{\beta}\left[\left\langle\left\langle\boldsymbol{u}_{\beta}\right\rangle^{\beta}\left\langle\boldsymbol{u}_{\beta}\right\rangle^{\beta}\right\rangle^{\beta}+\left\langle\left\langle\boldsymbol{u}_{\beta}\right\rangle^{\beta} \tilde{\boldsymbol{u}}_{\beta}\right\rangle^{\beta}+\left\langle\tilde{\boldsymbol{u}}_{\beta}\left\langle\boldsymbol{u}_{\beta}\right\rangle^{\beta}\right\rangle^{\beta}+\left\langle\tilde{\boldsymbol{u}}_{\beta} \tilde{\boldsymbol{u}}_{\beta}\right\rangle^{\beta}\right]\right) \\
& =\nabla \cdot\left(\epsilon_{\beta}\left[\left\langle\boldsymbol{u}_{\beta}\right\rangle^{\beta}\left\langle\boldsymbol{u}_{\beta}\right\rangle^{\beta}+\left\langle\boldsymbol{u}_{\beta}\right\rangle^{\beta}\left\langle\tilde{\boldsymbol{u}}_{\beta}\right\rangle^{\beta}+\left\langle\tilde{\boldsymbol{u}}_{\beta}\right\rangle^{\beta}\left\langle\boldsymbol{u}_{\beta}\right\rangle^{\beta}+\left\langle\tilde{\boldsymbol{u}}_{\beta} \tilde{\boldsymbol{u}}_{\beta}\right\rangle^{\beta}\right]\right) \\
& =\nabla \cdot\left(\epsilon_{\beta}\left[\left\langle\boldsymbol{u}_{\beta}\right\rangle^{\beta}\left\langle\boldsymbol{u}_{\beta}\right\rangle^{\beta}+\left\langle\tilde{\boldsymbol{u}}_{\beta} \tilde{\boldsymbol{u}}_{\beta}\right\rangle^{\beta}\right]\right) .
\end{aligned}
$$

The binomial terms in the second line of (A 8) are simplified by using the assumption of well-behaved volume averages (2.7). The second and third terms in the third line of (A 8) are approximated to zero, since the volume average of a subfilter scale quantity is small $\left(\left\langle\tilde{\boldsymbol{u}}_{\beta}\right\rangle^{\beta} \approx 0\right)$.

Equation (2.5) is applied to the first term on the right-hand side of (A $4 a$ ) to derive the pressure gradient in intrinsic quantities and subsequently the chain rule is applied, which yields

$$
-\frac{1}{\rho} \nabla\langle p\rangle=-\frac{1}{\rho} \nabla\left(\epsilon_{\beta}\langle p\rangle^{\beta}\right)=-\frac{1}{\rho}\left(\epsilon_{\beta} \nabla\langle p\rangle^{\beta}+\langle p\rangle^{\beta} \nabla \epsilon_{\beta}\right) .
$$


The viscous diffusion term on the right-hand side of (A $4 a)$ is rewritten in intrinsic quantities. Using the chain rule, this yields

$$
\begin{aligned}
v \nabla^{2}\left\langle\boldsymbol{u}_{\beta}\right\rangle & =v \nabla^{2}\left(\epsilon_{\beta}\left\langle\boldsymbol{u}_{\beta}\right\rangle^{\beta}\right) \\
& =v\left[\epsilon_{\beta} \nabla \cdot\left(\nabla\left\langle\boldsymbol{u}_{\beta}\right\rangle^{\beta}\right)+\nabla\left\langle\boldsymbol{u}_{\beta}\right\rangle^{\beta} \cdot \nabla \epsilon_{\beta}+\left\langle\boldsymbol{u}_{\beta}\right\rangle^{\beta} \nabla \cdot\left(\nabla \epsilon_{\beta}\right)+\nabla \epsilon_{\beta} \cdot \nabla\left\langle\boldsymbol{u}_{\beta}\right\rangle^{\beta}\right] \\
& =v \epsilon_{\beta} \nabla^{2}\left\langle\boldsymbol{u}_{\beta}\right\rangle^{\beta}+2 v \nabla\left\langle\boldsymbol{u}_{\beta}\right\rangle^{\beta} \cdot \nabla \epsilon_{\beta}+v\left\langle\boldsymbol{u}_{\beta}\right\rangle^{\beta} \nabla^{2} \epsilon_{\beta} .
\end{aligned}
$$

The intrinsic form of the third term on the right-hand side of (A $4 a)$ is derived in three steps. All transformation steps are given in (A 11):

$$
\begin{aligned}
v \nabla \cdot \int_{A_{\beta \sigma}} m \boldsymbol{u}_{\beta} \boldsymbol{n}_{\beta \sigma} \mathrm{d} A= & v \nabla \cdot \int_{A_{\beta \sigma}} m\left\langle\boldsymbol{u}_{\beta}\right\rangle^{\beta} \boldsymbol{n}_{\beta \sigma} \mathrm{d} A+v \nabla \cdot \int_{A_{\beta \sigma}} m \tilde{\boldsymbol{u}}_{\beta} \boldsymbol{n}_{\beta \sigma} \mathrm{d} A \\
= & v\left[\nabla\left\langle\boldsymbol{u}_{\beta}\right\rangle^{\beta}\right] \cdot \int_{A_{\beta \sigma}} m \boldsymbol{n}_{\beta \sigma} \mathrm{d} A+v\left\langle\boldsymbol{u}_{\beta}\right\rangle^{\beta} \cdot \nabla \int_{A_{\beta \sigma}} m \boldsymbol{n}_{\beta \sigma} \mathrm{d} A \\
& +v \nabla \cdot \int_{A_{\beta \sigma}} m \tilde{\boldsymbol{u}}_{\beta} \boldsymbol{n}_{\beta \sigma} \mathrm{d} A \\
= & -v \nabla\left\langle\boldsymbol{u}_{\beta}\right\rangle^{\beta} \cdot \nabla \epsilon_{\beta}-v\left\langle\boldsymbol{u}_{\beta}\right\rangle^{\beta} \cdot \nabla^{2} \epsilon_{\beta}+v \nabla \cdot \int_{A_{\beta \sigma}} m \tilde{\boldsymbol{u}}_{\beta} \boldsymbol{n}_{\beta \sigma} \mathrm{d} A .
\end{aligned}
$$

Firstly, the surface integral is decomposed according to Gray's decomposition. Secondly, the chain rule is applied to the intrinsic volume average term. In a third step we make use of the following geometrical theorem that follows from substituting $\psi_{\alpha}=1$ in $(2.3)$ :

$$
\int_{A_{\beta \sigma}} m \boldsymbol{n}_{\beta \sigma} \mathrm{d} A=-\nabla\langle 1\rangle .
$$

Note that the superficial average of one equals the porosity $\left(\langle 1\rangle=\epsilon_{\beta}\right)$.

We apply Gray's decomposition to the first term on the right-hand side of (A $4 a$ ), which yields

$$
\begin{aligned}
\int_{A_{\beta \sigma}} m\left[-\frac{p}{\rho} \boldsymbol{I}+v \nabla \boldsymbol{u}_{\beta}\right] \cdot \boldsymbol{n}_{\beta \sigma} \mathrm{d} A \\
=\int_{A_{\beta \sigma}} m\left[-\frac{\langle p\rangle^{\beta}}{\rho} \boldsymbol{I}+v \nabla\left\langle\boldsymbol{u}_{\beta}\right\rangle^{\beta}\right] \cdot \boldsymbol{n}_{\beta \sigma} \mathrm{d} A+\int_{A_{\beta \sigma}} m\left[-\frac{\tilde{p}}{\rho} \boldsymbol{I}+v \nabla \tilde{\boldsymbol{u}}_{\beta}\right] \cdot \boldsymbol{n}_{\beta \sigma} \mathrm{d} A \\
=\left[-\frac{\langle p\rangle^{\beta}}{\rho} \boldsymbol{I}+v \nabla\left\langle\boldsymbol{u}_{\beta}\right\rangle^{\beta}\right] \cdot \int_{A_{\beta \sigma}} m \boldsymbol{n}_{\beta \sigma} \mathrm{d} A+\int_{A_{\beta \sigma}} m\left[-\frac{\tilde{p}}{\rho} \boldsymbol{I}+v \nabla \tilde{\boldsymbol{u}}_{\beta}\right] \cdot \boldsymbol{n}_{\beta \sigma} \mathrm{d} A \\
=\left[-\frac{\langle p\rangle^{\beta}}{\rho} \boldsymbol{I}+v \nabla\left\langle\boldsymbol{u}_{\beta}\right\rangle^{\beta}\right] \cdot\left(-\nabla \epsilon_{\beta}\right)+\int_{A_{\beta \sigma}} m\left[-\frac{\tilde{p}}{\rho} \boldsymbol{I}+v \nabla \tilde{\boldsymbol{u}}_{\beta}\right] \cdot \boldsymbol{n}_{\beta \sigma} \mathrm{d} A \\
=\frac{\langle p\rangle^{\beta}}{\rho} \nabla \epsilon_{\beta}-v \nabla\left\langle\boldsymbol{u}_{\beta}\right\rangle^{\beta} \cdot \nabla \epsilon_{\beta}+\int_{A_{\beta \sigma}} m\left[-\frac{\tilde{p}}{\rho} \boldsymbol{I}+v \nabla \tilde{\boldsymbol{u}}_{\beta}\right] \cdot \boldsymbol{n}_{\beta \sigma} \mathrm{d} A .
\end{aligned}
$$

Since we assume that volume averages are well-behaved, we can further simplify the first integral term in the second line of (A 13) such that the geometrical theorem (A 12) can be applied to it. Also here we make use of $\langle 1\rangle=\epsilon_{\beta}$. 
A continuum model for flow driven by metachronally beating cilia

Substituting all terms (A 5)-(A 13) into (A 4a) yields

$$
\begin{aligned}
{\left[\left\langle\boldsymbol{u}_{\beta}\right\rangle^{\beta} \frac{\partial \epsilon_{\beta}}{\partial t}+\epsilon_{\beta} \frac{\partial\left\langle\boldsymbol{u}_{\beta}\right\rangle^{\beta}}{\partial t}\right]+\left[\left\langle\boldsymbol{u}_{\beta}\right\rangle^{\beta} \nabla \cdot\left\langle\boldsymbol{u}_{\beta}\right\rangle+\epsilon_{\beta}\left\langle\boldsymbol{u}_{\beta}\right\rangle^{\beta} \cdot \nabla\left\langle\boldsymbol{u}_{\beta}\right\rangle^{\beta}\right] } \\
+\left[\nabla \cdot\left(\epsilon_{\beta}\left[\left\langle\boldsymbol{u}_{\beta}\right\rangle^{\beta}\left\langle\boldsymbol{u}_{\beta}\right\rangle^{\beta}+\langle\tilde{\boldsymbol{u}} \tilde{\boldsymbol{u}}\rangle^{\beta}\right]\right)\right]-\left[\nabla \cdot\left(\epsilon_{\beta}\left\langle\boldsymbol{u}_{\beta}\right\rangle^{\beta}\left\langle\boldsymbol{u}_{\beta}\right\rangle^{\beta}\right)\right] \\
=-\left[\frac{1}{\rho} \epsilon_{\beta} \nabla\langle p\rangle^{\beta}+\frac{1}{\rho}\langle p\rangle^{\beta} \nabla \epsilon_{\beta}\right] \\
+\left[v \epsilon_{\beta} \nabla^{2}\left\langle\boldsymbol{u}_{\beta}\right\rangle^{\beta}+2 v \nabla\left\langle\boldsymbol{u}_{\beta}\right\rangle^{\beta} \cdot \nabla \epsilon_{\beta}+v\left\langle\boldsymbol{u}_{\beta}\right\rangle^{\beta} \nabla^{2} \epsilon_{\beta}\right] \\
+\left[-v \nabla \epsilon_{\beta} \cdot \nabla\left\langle\boldsymbol{u}_{\beta}\right\rangle^{\beta}-v\left\langle\boldsymbol{u}_{\beta}\right\rangle^{\beta} \nabla^{2} \epsilon_{\beta}+v \nabla \cdot \int_{A_{\beta \sigma}} m \tilde{\boldsymbol{u}}_{\beta} \boldsymbol{n}_{\beta \sigma} \mathrm{d} A\right] \\
+\left[\frac{\langle p\rangle^{\beta}}{\rho} \nabla \epsilon_{\beta}-v \nabla\left\langle\boldsymbol{u}_{\beta}\right\rangle^{\beta} \cdot \nabla \epsilon_{\beta}+\int_{A_{\beta \sigma}} m\left(-\frac{\tilde{p}}{\rho} \boldsymbol{I}+v \nabla \tilde{\boldsymbol{u}}_{\beta}\right) \cdot \boldsymbol{n}_{\beta \sigma} \mathrm{d} A\right] .
\end{aligned}
$$

Nine terms of (A 14) cancel each other out, yielding

$$
\begin{gathered}
{\left[\left\langle\boldsymbol{u}_{\beta}\right\rangle^{\beta} \frac{\partial \epsilon_{\beta}}{\partial t}+\epsilon_{\beta} \frac{\partial\left\langle\boldsymbol{u}_{\beta}\right\rangle^{\beta}}{\partial t}\right]+\left[\left\langle\boldsymbol{u}_{\beta}\right\rangle^{\beta} \nabla \cdot\left\langle\boldsymbol{u}_{\beta}\right\rangle+\epsilon_{\beta}\left\langle\boldsymbol{u}_{\beta}\right\rangle^{\beta} \cdot \nabla\left\langle\boldsymbol{u}_{\beta}\right\rangle^{\beta}\right]} \\
+\left[\nabla \cdot \epsilon_{\beta}\langle\tilde{\boldsymbol{u}} \tilde{\boldsymbol{u}}\rangle^{\beta}\right]=-\left[\frac{1}{\rho} \epsilon_{\beta} \nabla\langle p\rangle^{\beta}\right]+\left[v \epsilon_{\beta} \nabla^{2}\left\langle\boldsymbol{u}_{\beta}\right\rangle^{\beta}\right] \\
+v \boldsymbol{\nabla} \cdot \int_{A_{\beta \sigma}} m \tilde{\boldsymbol{u}}_{\beta} \boldsymbol{n}_{\beta \sigma} \mathrm{d} A+\int_{A_{\beta \sigma}} m\left(-\frac{\tilde{p}}{\rho} \boldsymbol{I}+v \nabla \tilde{\boldsymbol{u}}_{\beta}\right) \cdot \boldsymbol{n}_{\beta \sigma} \mathrm{d} A .
\end{gathered}
$$

The sum of the first and third terms of (A 15) equals zero due to mass conservation according to $(2.4 a$ ). Finally we divide (A 15) by the porosity of the solid phase, and write it together with (A 3) to derive the VANS equations in intrinsic quantities in the form as given in $(2.8 a)$ and $(2.8 b)$ :

$$
\begin{gathered}
\nabla \cdot\left\langle\boldsymbol{u}_{\beta}\right\rangle^{\beta}=-\frac{1}{\epsilon_{\beta}}\left(\frac{\partial \epsilon_{\beta}}{\partial t}+\left\langle\boldsymbol{u}_{\beta}\right\rangle^{\beta} \cdot \nabla \epsilon_{\beta}\right), \\
\frac{\partial\left\langle\boldsymbol{u}_{\beta}\right\rangle^{\beta}}{\partial t}+\left\langle\boldsymbol{u}_{\beta}\right\rangle^{\beta} \cdot \nabla\left\langle\boldsymbol{u}_{\beta}\right\rangle^{\beta}+\frac{1}{\epsilon_{\beta}} \nabla \cdot \epsilon_{\beta}\left\langle\tilde{\boldsymbol{u}}_{\beta} \tilde{\boldsymbol{u}}_{\beta}\right\rangle^{\beta} \\
=-\frac{1}{\rho} \nabla\left\langle p_{\beta}\right\rangle^{\beta}+v \nabla^{2}\left\langle\boldsymbol{u}_{\beta}\right\rangle^{\beta}+\frac{v}{\epsilon_{\beta}} \nabla \cdot \int_{A_{\beta \sigma}} m \tilde{\boldsymbol{u}}_{\beta} \boldsymbol{n}_{\beta \sigma} \mathrm{d} A \\
+\frac{1}{\epsilon_{\beta}} \int_{A_{\beta \sigma}} m\left(-\frac{\tilde{p}_{\beta}}{\rho} \boldsymbol{I}+v \nabla \tilde{\boldsymbol{u}}_{\beta}\right) \cdot \boldsymbol{n}_{\beta \sigma} \mathrm{d} A .
\end{gathered}
$$

\section{Appendix B. Derivation of the bulk porosity}

An analytical expression for the porosity $\epsilon_{\beta}$ of the solid phase is derived. The porosity $\epsilon_{\beta}$ is generally defined as the void fraction or fluid volume fraction:

$$
\epsilon_{\beta}=\frac{V_{\beta}}{V}=1-\epsilon_{\sigma}=1-\frac{V_{\sigma}}{V} .
$$




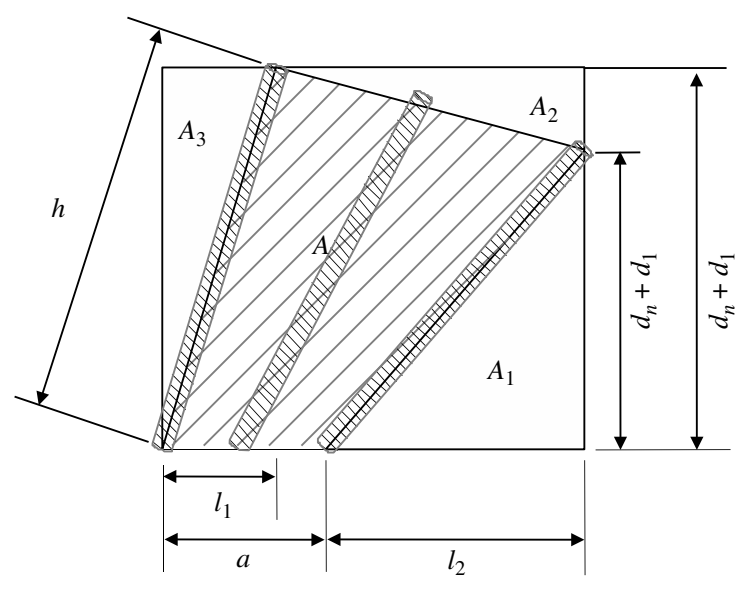

FIGURE 12. Representative unit cell volume bordered by the centreline axes of four neighbouring cilia. Partial areas $A_{1}$ to $A_{3}$ are used to compute area $A$ of the unit cell.

For simplicity the porosity inside the cilia layer is defined as the volume fraction of a representative volume $V=A b$ whereas $A$ is indicated in figure 12 and $b$ is the cilia spacing in the $z$-direction. The resulting porosity distribution varies only along the $x$-direction but stays constant over the height of the cilia layer. This approximation holds if $A a / \lambda^{2} \ll 1$. The representative volume as shown in figure 12 is bordered by the centreline axes of four neighbouring cilia. For simplicity it is assumed that the tip of the fifth cilium in the middle of the volume lies on the surface of the representative volume $V$ such that the solid phase $V_{\sigma}$ can be approximated to two times the volume of a cilium. We determine the volume $V$ by simple geometrical relations (see figure 12),

$$
\begin{aligned}
V_{\sigma} & =\frac{1}{2} h \pi D^{2}, \\
V & =A b=b\left[A_{\text {total }}-A_{1}-A_{2}-A_{3}\right],
\end{aligned}
$$

whereas $A_{\text {total }}$ denotes the sum of all partial areas:

$$
\begin{aligned}
A_{\text {total }} & =\left(a+l_{2}\right)\left(d_{1}+d_{n}\right), \\
A_{1} & =\frac{1}{2} l_{2}\left(d_{2}+d_{n}\right), \\
A_{2} & =\frac{1}{2}\left(a+l_{2}-l_{1}\right)\left(d_{1}-d_{2}\right), \\
A_{3} & =\frac{1}{2} l_{1}\left(d_{1}+d_{n}\right) .
\end{aligned}
$$

Substituting (B 2a), (B 2b) and (B 3a)-(B 4) into (B 1) leads to the bulk porosity inside the cilia layer as given by (3.6):

$$
\epsilon_{\beta, c}(x, t)=1-\frac{\pi D^{2} h}{b\left(a\left[d_{1}+d_{2}+2 d_{n}\right]-l_{1}\left[d_{2}+d_{n}\right]+l_{2}\left[d_{1}+d_{n}\right]\right)} .
$$

\section{REFERENCES}

Baltussen, M., Anderson, P., Bosab, F. \& Den Toonder, J. 2009 Inertial flow effects in a micro-mixer based on artificial cilia. Lab on a Chip 9, 2326-2331. 
BARLOW, D. \& SLEIGH, M. A. 1993 Water propulsion speeds and power output by comb plates of the ctenophore Pleurobrachia pileus under different conditions. J. Expl Biol. 183, 149-163.

BARTON, C. \& RAYNOR, S. 1967 Analytical investigation of cilia induced mucous flow. Bull. Math. Biophys. 29, 419-428.

Bird, R. B., Stewart, W. E. \& Lightfoot, E. N. 2002 Transport Phenomena. Wiley.

BLAKE, J. R. 1972 Model for micro-structure in ciliated organisms. J. Fluid Mech. 55, 1-23.

BLAKE, J. R. \& WinET, H. 1980 On the mechanics of muco-ciliary transport. Biorheology 17, $125-134$.

BRENNEN, C. 1974 Oscillating-boundary-layer theory for ciliary propulsion. J. Fluid Mech. 65, 799-824.

Brennen, C. \& Winet, H. 1977 Fluid-mechanics of propulsion by cilia and flagella. Annu. Rev. Fluid Mech. 9, 339-398.

Breugem, W.-P. 2007 The effective viscosity of a channel-type porous medium. Phys. Fluids 19, 103104.

BReugem, W.-P. \& Boersma, B. J. 2005 Direct numerical simulations of turbulent flow over a permeable wall using a direct and a continuum approach. Phys. Fluids 17, 025103.

Breugem, W.-P., Boersma, B. J. \& UittenbogaArd, R. E. 2006 The influence of wall permeability on turbulent channel flow. J. Fluid Mech. 562, 35-72.

Dauptain, A., Favier, J. \& Bottaro, A. 2008 Hydrodynamics of ciliary propulsion. J. Fluids Struct. 24, 1156-1165.

FAhrni, F., Prins, M. \& VAN IJZEndoorn, L. 2009 Micro-fluidic actuation using magnetic artificial cilia. Lab on a Chip 9, 3413-3421.

GRAY, W. G. 1975 A derivation of the equations for multi-phase transport. Chem. Engng Sci. 30, 229-233.

Gueron, S. \& Levit-Gurevich, K. 1998 Computation of the internal forces in cilia: application to ciliary motion, the effects of viscosity, and cilia interactions. Biophys. J. 74, 1658-1676.

Gueron, S., Levit-Gurevich, K., Liron, N. \& Blum, J. J. 1997 Cilia internal mechanism and metachronal coordination as the result of hydrodynamical coupling. Proc. Natl Acad. Sci. USA 94, 6001-6006.

GuERON, S. \& LIRON, N. 1993 Simulations of three-dimensional ciliary beats and cilia interactions. Biophys. J. 65, 499-507.

Guirao, B. \& JoAnny, J. F. 2007 Spontaneous creation of macroscopic flow and metachronal waves in an array of cilia. Biophys. J. 92, 1900-1917.

Heys, J. J., Gedeon, T., Knott, B. C. \& Kim, Y. 2008 Modeling arthropod filiform hair motion using the penalty immersed boundary method. J. Biomech. 41, 977-984.

Hussong, J., Schorr, N., Belardi, J., Prucker, O., Rühe, J. \& Westerweel, J. 2011 Experimental investigation of the flow induced by artificial cilia. Lab on a Chip 11, 2017-2022.

KATZ, D. F. 1974 Propulsion of microorganisms near solid boundaries. J. Fluid Mech. 64, 33-49.

Khaderi, S. N., Baltussen, M. G. H. M., Anderson, P. D., IoAn, D., Den Toonder, J. M. J. \& ONCK, P. R. 2009 Nature-inspired microfluidic propulsion using magnetic actuation. Phys. Rev. E 79, 046304.

Khaderi, S. N., Baltussen, M. G. H. M., Anderson, P. D., Den Toonder, J. M. J. \& Onck, P. R. 2010 Breaking of symmetry in microfluidic propulsion driven by artificial cilia. Phys. Rev. E 82, 027302.

Khatavkar, V. V., Anderson, P. D., Den Toonder, J. M. J. \& Meijer, H. E. H. 2007 Active micromixer based on artificial cilia. Phys. Fluids 19, 0836059.

KIM, Y. \& PESKIN, C. S. 2007 Penalty immersed boundary method for an elastic boundary with mass. Phys. Fluids 19, 053103.

King, M., Agarwal, M. \& Shukla, J. B. 1993 A planar model for mucociliary transport: effect of mucus viscoelasticity. Biorheology 30, 49-61.

Lardner, T. J. \& ShaCK, W. J. 1972 Cilia transport. Bull. Math. Biophys. 34, 325-335.

Lenz, P. \& Ryskin, A. 2006 Collective effects in ciliar arrays. Phys. Biol. 3, 285-294. 
LIRON, N. 1978 Fluid transport by cilia between parallel plates. J. Fluid Mech. 86, 705-726.

LiRON, N. \& MOCHON, S. 1976 Discrete-cilia approach to propulsion of ciliated microorganisms. J. Fluid Mech. 75, 593-607.

MacDonald, I. F., Elsayed, M. S., Mow, K. \& Dullien, F. A. L. 1979 Flow through porous media: the Ergun equation revisited. Ind. Engng Chem. Fundam. 18, 199-208.

Merz, R. A. \& EdWARDS, D. R. 1998 Jointed setae: their role in locomotion and gait transitions in polychaete worms. J. Exp. Mar. Biol. Ecol. 228, 273-290.

Mitran, S. M. 2007 Metachronal wave formation in a model of pulmonary cilia. Comput. Struct. 85, 763-774.

Nielsen, N. F. \& LARSEN, P. S. 1993 A note on ciliated plane channel flow with a pressure gradient. J. Fluid Mech. 257, 97-110.

Nonaka, S., Yoshiba, S., Watanabe, D., Ikeuchi, S., Goto, T., Marshall, W. F. \& HAMADA, H. 2005 De novo formation of left-right asymmetry by posterior tilt of nodal cilia. PLOS Biol. 3, e268.

OH, K., Chung, J.-H., Devasia, S. \& Riley, J. J. 2009 Bio-mimetic silicone cilia for microfluidic manipulation. Lab on a Chip 9, 1561-1566.

PESKIN, C. S. 1972 Flow patterns around heart valves: a numerical method. J. Comput. Phys. 10, 252-271.

PurCell, E. M. 1977 Life at low Reynolds number. Am. J. Phys. 45, 3-11.

QuintARD, M. \& WhitAKER, S. 1994 Transport in ordered and disordered porous media I: the cellular average and the use of weighting functions. Trans. Porous Med. 14, 163-177.

Shields, A. R., Fiser, B. L., Evans, B. A., Falvo, M. R., Washburn, S. \& Superfine, R. 2010 Biomimetic cilia arrays generate simultaneous pumping and mixing regimes. Proc. Natl Acad. Sci. 107 (36), 15670-15675.

Singh, H., LAibinis, P. E. \& Hatton, T. A. 2005 Synthesis of flexible magnetic nanowires of permanently linked core-shell magnetic beads tethered to a glass surface patterned by microcontact printing. Nano Lett. 5, 2149-2154.

Smith, D. J., Gaffney, E. A. \& Blake, J. R. 2007 a A viscoelastic traction layer model of muco-ciliary transport. Bull. Math. Biol. 69, 289-327.

Smith, D. J., GaffNey, E. A. \& Blake, J. R. $2007 b$ Discrete cilia modelling with singularity distributions: application to the embryonic node and the airway surface liquid. Bull. Math. Biol. 69, 1477-1510.

Smith, D. J., Gaffney, E. A. \& Blake, J. R. 2008 Modelling mucociliary clearance. Respir. Physiol. Neurobiol. 163, 178-188.

TAYLOR, G. 1951 Analysis of the swimming of microscopic organisms. Proc. R. Soc. Lond. A Mat. 209, 447-461.

den Toonder, J., Bos, F., Broer, D., Filippini, L., Gillies, M., de Goede, J., Mol, T., Reijme, M., Talen, W., Wilderbeek, H., KhataVkar, V. \& Anderson, P. 2007 Artificial cilia for active micro-fluidic mixing. Lab on a Chip 8, 533-541.

TUCK, E. O. 1968 A note on a swimming problem. J. Fluid Mech. 31, 305-308.

Uhlmann, M. 2005 An immersed boundary method with direct forcing for the simulation of particulate flows. J. Comput. Phys. 209, 448-476.

Vilfan, M., Potocnik, A., Kavcic, B., Osterman, N., Poberjaj, I., Vilfan, A. \& Babic, D. 2009 Self-assembled artificial cilia. Proc. Natl Acad. Sci. 5 (5), 1844-1847.

Wesseling, P. 2001 Principles of Computational Fluid Dynamics. Springer.

Whitaker, S. 1996 The Forchheimer equation: a theoretical development. Transp. Porous Med. 25, 27-61.

WhitaKer, S. 1999 The Method of Volume Averaging. Kluwer.

Zhou, Z. G. \& LIU, Z. W. 2008 Biomimetic cilia based on MEMS technology. J. Bionic Engng 5, $358-365$. 